\title{
Highly Enantioselective Insertion of Carbenoids into N-H Bonds Catalyzed by Copper Complexes of Chiral Spiro Bisoxazolines
}

\author{
Bin Liu, Shou-Fei Zhu, Wei Zhang, Chao Chen, Qi-Lin Zhou* \\ State Key Laboratory and Institute of Elemento-organic Chemistry, Nankai University \\ Tianjin 300071, China.
}

\section{CONTENTS:}

1. Preparation of Chiral Spiro Bisoxazoline Ligands

2. General Procedure for Cu-Catalyzed Asymmetric Insertion of Carbenoid into Anilines

$\begin{array}{lll}\text { 3. Analytical Data for N-H Insertion Products } & \text { S8 }\end{array}$

4. NMR Spectra for New Ligands and Intermediates S18

5. NMR Spectra for New N-H Insertion Products S25

6. HPLC Charts for N-H Insertion Products S33

General. The reactions and manipulations were performed under an atmosphere of argon by using standard Schlenk techniques. Anhydrous dichloromethane, chloroform, acetonitrile and DMSO were distilled from calcium hydride. Anhydrous benzene and THF was distilled from sodium benzophenone ketyl prior to use. The aniline and substituted anilines were purified by distillation or recrystallization before use. The bis(triflate) 5 was synthesized according to the previous method. ${ }^{1} \mathrm{CuCl}$ and $\mathrm{CuPF}_{6}(\mathrm{MeCN})_{4}$ were prepared according to the literature

\footnotetext{
${ }^{1}$ Liu, B.; Zhu, S.-F.; Wang, L.-X.; Zhou, Q.-L. Tetrahedron: Asymmetry 2006, 17, 634.
} 
method. ${ }^{2}$ NaBARF. $\left(\mathrm{H}_{2} \mathrm{O}\right)_{1.5}$ was prepared according to the literatures. ${ }^{3} \mathrm{CuOTf}(\text { toluene })_{1 / 2}$, $\mathrm{CuBr}\left(\mathrm{Me}_{2} \mathrm{~S}\right), \mathrm{CuCl}_{2}, \mathrm{Cu}(\mathrm{OTf})_{2}, \mathrm{Pd}(\mathrm{OAc})_{2}$, dppp and HOBt were purchased from Aldrich or Acros company and used without further purification. NMR spectra were recorded with a Bruker or Varian spectrometer at 400 or $300\left({ }^{1} \mathrm{H}\right.$ NMR $), 100$ or $75\left({ }^{13} \mathrm{C}\right.$ NMR $) \mathrm{MHz}$ with $\mathrm{CDCl}_{3}$ as solvent. Chemical shifts were reported in ppm down field from internal $\mathrm{Me}_{4} \mathrm{Si}$. HRMS were recorded on VG ZAB-HS mass spectrometer with EI resource. Optical rotations were measured using a Perkin Elmer Model 341 polarimeter. HPLC analyses were performed using a Hewlett Packard Model HP 1100 Series chromatography.

\section{Preparation of Chiral Spiro Bisoxazoline Ligands 1}
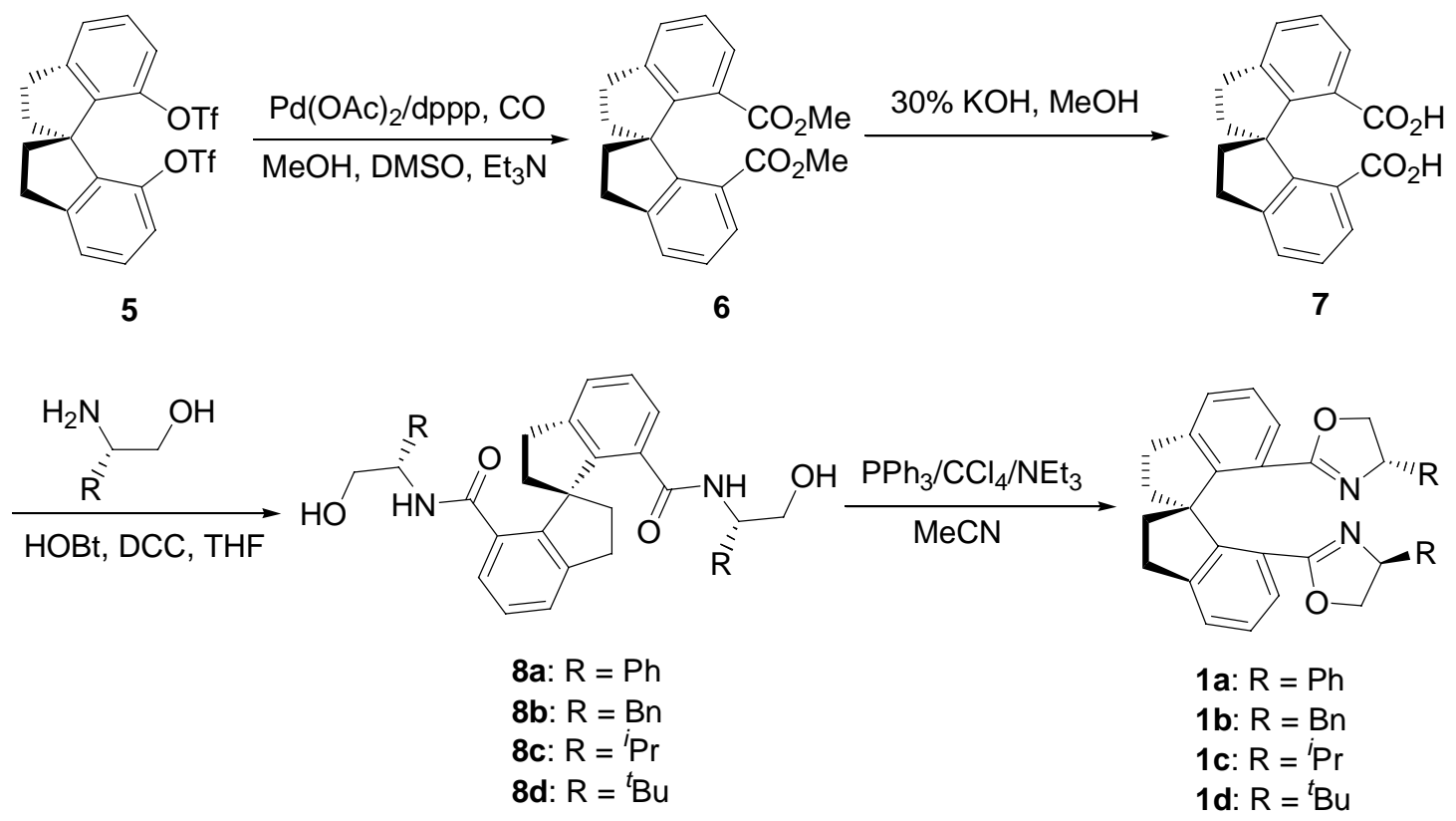

Synthesis of (S)-1,1'-spirobiindanyl-7,7'-dicarboxylic acid dimethyl ester [(S)-6]

\footnotetext{
2 Inorg. Synth. Vol. II, p. 1

3 (a) Brookhart, M.; Grant, B.; Volpe, A. F. Organometallics 1992, 11, 3920. (b) Nishida, H.; Takada, N.; Yoshimura, M.; Sonoda, T.; Kobayashi, H. Bull. Chem. Soc. Jpn. 1984, 57, 2600.
} 


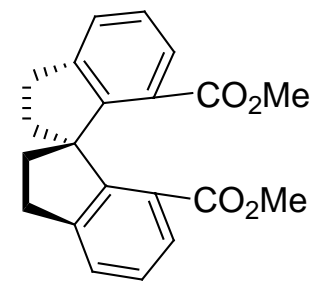

A mixture of bis(triflate) $(S)-5(5.2 \mathrm{~g} 10 \mathrm{mmol}), \operatorname{Pd}(\mathrm{OAc})_{2}(225 \mathrm{mg}, 1.0 \mathrm{mmol})$, 1,3-bis(diphenylphosphino)propane (dppp, $412 \mathrm{mg}, 1.0 \mathrm{mmol}), \mathrm{MeOH}(60 \mathrm{~mL})$, DMSO (90 $\mathrm{mL})$ and $\mathrm{Et}_{3} \mathrm{~N}(24 \mathrm{~mL})$ was saturated with $\mathrm{CO}$ and stirred under $\mathrm{CO}$ atmosphere at $70{ }^{\circ} \mathrm{C}$ for full conversion. After cooling to room temperature, the reaction mixture was concentrated in reduced pressure. The residue was purified by flash chromatography (ethyl acetate/petroleum ether $=1: 8)$ to afford product $(S)-6(2.85 \mathrm{~g}, 85 \%)$ as a white solid. Mp $118-120{ }^{\circ} \mathrm{C} ;[\alpha]^{20}{ }_{\mathrm{D}}=$ -278 (c 1.0, $\left.\mathrm{CHCl}_{3}\right) ;{ }^{1} \mathrm{H}$ NMR $\delta$ 2.27-2.32 (m, 2H), 2.53-2.61 (m, 2H), 3.00-3.11 (m, 4H), $3.16(\mathrm{~s}, 6 \mathrm{H}), 7.23(\mathrm{t}, J=7.6 \mathrm{~Hz}, 2 \mathrm{H}), 7.43(\mathrm{~d}, J=7.2 \mathrm{~Hz}, 2 \mathrm{H}), 7.60(\mathrm{~d}, J=7.6 \mathrm{~Hz}, 2 \mathrm{H}) ;{ }^{13} \mathrm{C}$ NMR $\delta 30.9,38.3,51.3,63.3,126.5,126.9,128.3,128.7,145.3,149.7,167.9$; HRMS (EI) Calcd for $\mathrm{C}_{21} \mathrm{H}_{20} \mathrm{O}_{4}$ : 336.1362; Found: 336.1367 .

\section{Synthesis of $(S)-1,1^{\prime}$-spirobiindanyl-7,7'-dicarboxylic acid [(S)-7] ${ }^{1}$}

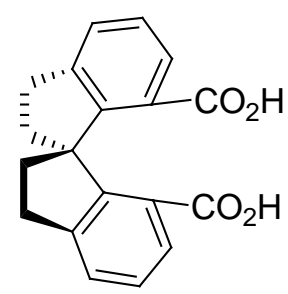

The compound (S)-6 (3.1 g, $9.2 \mathrm{mmol})$ was added to a solution of $\mathrm{MeOH}(40 \mathrm{~mL})$ and $30 \%$ aqueous $\mathrm{KOH}(40 \mathrm{~mL})$ and stirred at refluxing for $10 \mathrm{~h}$. The resulting mixture was cooled to room temperature, diluted with water $(200 \mathrm{~mL})$ and adjusted $\mathrm{pH}$ to $4 \sim 5$ with $6 \mathrm{M} \mathrm{HCl}$, and extracted with ethyl acetate $(120 \mathrm{~mL})$ for three times. The combined organic layer was washed with water and saturated brine, dried over anhydrous $\mathrm{MgSO}_{4}$, and concentrated under reduced pressure. The residue was purified by flash chromatography (ethyl acetate/petroleum ether $=1: 1)$ to afford product $(S)-7(2.8 \mathrm{~g}, 97 \%)$. Further purification by recrystallization 
from toluene afforded white solid. $\mathrm{Mp}=230-231{ }^{\circ} \mathrm{C} ;{ }^{1} \mathrm{H}$ NMR $\delta 2.22-2.27(\mathrm{~m}, 2 \mathrm{H})$, $2.43-2.51(\mathrm{~m}, 2 \mathrm{H}), 2.97-3.13(\mathrm{~m}, 4 \mathrm{H}), 7.12(\mathrm{t}, J=8.0 \mathrm{~Hz}, 2 \mathrm{H}), 7.39(\mathrm{~d}, J=7.2 \mathrm{~Hz}, 2 \mathrm{H}), 7.62$ $(\mathrm{d}, J=7.6 \mathrm{~Hz}, 2 \mathrm{H}), 10.8$ (brs, 2H).

Synthesis of $\left(S_{a}, S, S\right)-7,7^{\prime}$-bis[N-(2-hydroxy-1-phenylethyl)amide]-1,1'-spirobiindane $\left[\left(S_{a}, S, S\right)-8 a\right]^{1}$

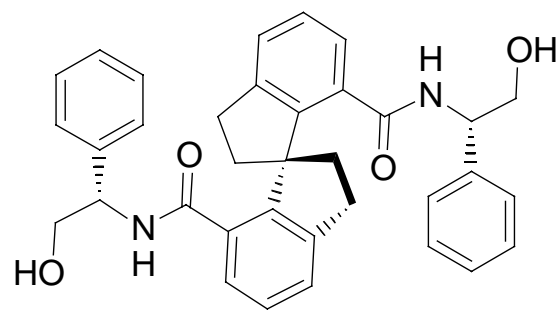

A solution of $(S)-7$ (308 mg, $1.0 \mathrm{mmol})$, dicyclohexyl carbodiimide (870 mg, $4.2 \mathrm{mmol})$, benzotriazol-1-ol (300 mg, $2.2 \mathrm{mmol})$ and (S)-2-amino-2-phenylethanol (300 mg, $2.2 \mathrm{mmol}$ ) in dry THF was cooled to $-5{ }^{\circ} \mathrm{C}$ and stirred for 1 hour. After spontaneously warmed to room temperature the mixture was stirred for over night. The resulting mixture was concentrated under reduced pressure, and the residue was purified by flash chromatography (ethyl acetate) to afford $\left(S_{a}, S, S\right)-8 \mathbf{a}(475 \mathrm{mg}, 87 \%)$ as a white solid. Mp $104-107^{\circ} \mathrm{C} ;[\alpha]^{20}{ }_{\mathrm{D}}-103$ (c 0.5 , $\left.\mathrm{CHCl}_{3}\right) ;{ }^{1} \mathrm{H}$ NMR (400 MHz, $\left.\mathrm{CDCl}_{3}\right): \delta .1 .22-1.30$ (m, 2H), 2.23-2.29 (m, 2H), 2.44-2.50 (m, 2H), $2.86(\mathrm{~s}, 2 \mathrm{H}), 2.94-3.02(\mathrm{~m}, 4 \mathrm{H}), 3.50-3.61(\mathrm{~m}, 4 \mathrm{H}), 4.42-4.46(\mathrm{~m}, 2 \mathrm{H}), 6.91-6.99(\mathrm{~m}$, $6 \mathrm{H}), 7.14(\mathrm{t}, J=7.6 \mathrm{~Hz}, 2 \mathrm{H}), 7.24-7.30(\mathrm{~m}, 8 \mathrm{H})$.

Synthesis of $\left(S_{a}, S, S\right)-7,7^{\prime}$-bis[N-(1-benzyl-2-hydroxyethyl)amide]-1,1'-spirobiindane $\left[\left(S_{a}, S, S\right)-8 b\right]$

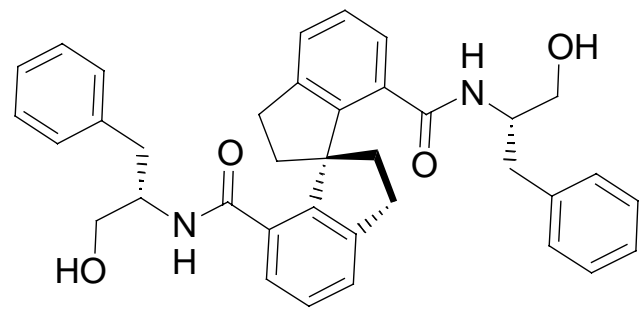


The compound $\left(S_{a}, S, S\right)$-8b was synthesized from (S)-2-amino-3-phenylpropan-1-ol in 99\% yield by the same procedure as that for $\left(S_{a}, S, S\right)-\mathbf{8 a}$. White solid, mp $175-178{ }^{\circ} \mathrm{C} ;[\alpha]^{20}{ }_{\mathrm{D}}=$ -106 (c 1.0, $\left.\mathrm{CHCl}_{3}\right)$; ${ }^{1} \mathrm{H}$ NMR: $\delta$ 2.23-2.27 (m, 2H), 2.35-2.49 (m, 6H), 2.98-3.00 (m, 6H), 3.18-3.28 (m, 4H), 3.68-3.69 (m, 2H), $6.90(\mathrm{~d}, J=7.2 \mathrm{~Hz}, 2 \mathrm{H}), 7.01(\mathrm{~d}, J=7.8 \mathrm{~Hz}, 2 \mathrm{H})$, $7.08(\mathrm{~d}, J=7.2 \mathrm{~Hz}, 3 \mathrm{H}), 7.15-7.19(\mathrm{~m}, 4 \mathrm{H}), 7.22-7.28(\mathrm{~m}, 5 \mathrm{H}) ;{ }^{13} \mathrm{C}$ NMR: $\delta 30.7,26.1,40.3$, $53.9,62.2,63.4,126.3,126.7,127.4,128.7,129.3$, 133.9, 138.2, 145.54, 170.5; HRMS (EI) Calcd for $\mathrm{C}_{37} \mathrm{H}_{38} \mathrm{~N}_{2} \mathrm{O}_{4}$ : 574.2832; Found: 574.2834.

Synthesis of $\left(S_{a}, S, S\right)-7,7^{\prime}$-bis[N-(1-hydroxymethyl-2-methylpropyl)amide]-1,1'-spirobiindane $\left[\left(S_{a}, S, S\right)-8 \mathrm{c}\right]$

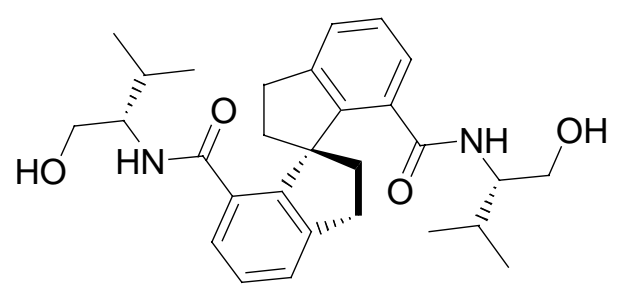

The compound $\left(S_{a}, S, S\right)-8 \mathrm{c}$ was synthesized from (S)-2-amino-3-methylbutan-1-ol in 99\% yield by the same procedure as that for $\left(S_{a}, S, S\right)-8 \mathbf{a}$. White solid, mp $166-170{ }^{\circ} \mathrm{C} ;[\alpha]^{20}{ }_{\mathrm{D}}=$ -126 (c 1.0, $\left.\mathrm{CHCl}_{3}\right)$; ${ }^{1} \mathrm{H}$ NMR: $\delta 0.81(\mathrm{t}, J=6.9 \mathrm{~Hz}, 12 \mathrm{H}), 1.63-1.67(\mathrm{~m}, 2 \mathrm{H}), 2.28-2.34(\mathrm{~m}$, 2H), 2.44-2.48 (m, 2H), 2.99-3.04 (m, 6H), 3.13-3.16 (m, 2H), 3.24-3.35 (m, 4H), 6.85 (d, $2 \mathrm{H}, J=6.9 \mathrm{~Hz}), 7.17-7.32(\mathrm{~m}, 6 \mathrm{H}) ;{ }^{13} \mathrm{C} \mathrm{NMR}: \delta 18.9,19.4,28.6,30.5,40.1,58.9,63.2$, 126.0, 126.3, 127.3, 134.2, 145.1, 171.0; HRMS (EI) Calcd for $\mathrm{C}_{29} \mathrm{H}_{38} \mathrm{~N}_{2} \mathrm{O}_{4}$ : 478.2832; Found: 478.2839 .

Synthesis of $\left(S_{a}, S, S\right)-7,7^{\prime}$-bis[N-(2-hydroxy-1-tert-butylethyl)amide]-1,1'-spirobiindane $\left[\left(S_{a}, S, S\right)-8 \mathrm{~d}\right]$ 


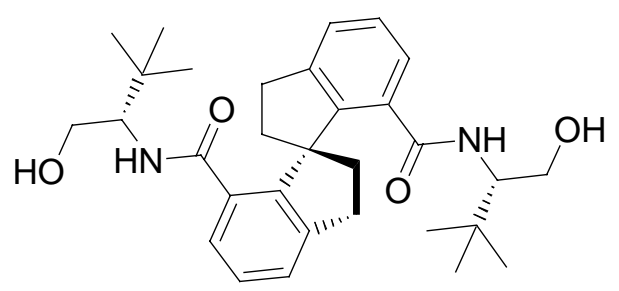

The compound $\left(S_{a}, S, S\right)$-8d was synthesized from $(S)$-2-amino-3,3-dimethylbutan-1-ol in 99\% yield by the same procedure as that for $\left(S_{a}, S, S\right)-8 \mathbf{a}$. White solid, mp $200-203{ }^{\circ} \mathrm{C},[\alpha]^{20}{ }_{\mathrm{D}}=$ -66.4 (c 1.0, $\left.\mathrm{CHCl}_{3}\right) ;{ }^{1} \mathrm{H}$ NMR: $\delta 0.79$ (s, 18H), 2.24-2.31 (m, 2H), 2.44-2.52 (m, 2H), 2.71 (brs, 2H), 2.98-3.03 (m, 4H), 3.28-3.33 (m, 4H), 3.45-3.52 (m, 2H), 6.70 (d, J=6.8 Hz, 2H), 7.22-7.29 (m, 6H); ${ }^{13} \mathrm{C}$ NMR: $\delta 27.1,30.7,33.9,40.3,61.5,63.0,126.3,126.7,126.5,134.6$, 145.3, 171.3; HRMS (EI) Calcd for $\mathrm{C}_{31} \mathrm{H}_{42} \mathrm{~N}_{2} \mathrm{O}_{4}$ : 506.3145; Found: 506.3136.

\section{Synthesis of $\left(S_{a}, S, S\right)-7,7^{\prime}$-bis[4-phenyloxazolin-2-yl]-1,1'-spirobiindane [( $\left.\left.S_{a} S, S\right)-1 \mathrm{a}\right]{ }^{1}$}

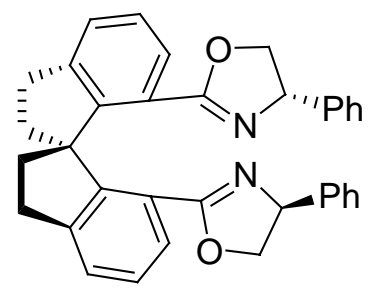

A solution of $\left(S_{a}, S, S\right)-8 \mathbf{a}(234 \mathrm{mg}, 0.42 \mathrm{mmol})$, triphenylphosphine (440 $\left.\mathrm{mg}, 1.68 \mathrm{mmol}\right)$, triethylamine $(170 \mathrm{mg}, 1.68 \mathrm{mmol})$, tetrachloromethane $(260 \mathrm{mg}, 1.68 \mathrm{mmol})$ in dry acetonitrile was stirred over night at room temperature. After concentrated in vacuum, the residue was dissolved in $\mathrm{CH}_{2} \mathrm{Cl}_{2}$, washed with water, dried over anhydrous magnesium sulfate, and then concentrated in vacuum. The crude product was purified by chromatography on silica gel column (ethyl acetate/petroleum ether $=1: 3)$ to afford $\left(S_{a}, S, S\right)-\mathbf{1 a}(200 \mathrm{mg}, 93 \%)$ as a white solid. Mp $167-169^{\circ} \mathrm{C} ;[\alpha]^{20}{ }_{\mathrm{D}}-321\left(c\right.$ 0.5, $\left.\mathrm{CH}_{2} \mathrm{Cl}_{2}\right) ;{ }^{1} \mathrm{H}$ NMR $\left(300 \mathrm{MHz}, \mathrm{CDCl}_{3}\right): \delta$ $2.31(\mathrm{dd}, J=4.8$ and $7.2 \mathrm{~Hz}, 2 \mathrm{H}), 2.60$ (q, $J=10.8 \mathrm{~Hz}, 2 \mathrm{H}), 2.98-3.16(\mathrm{~m}, 4 \mathrm{H}), 3.34-3.41$ (m, 2H), $3.72(\mathrm{t}, J=7.2 \mathrm{~Hz}, 2 \mathrm{H}), 4.98(\mathrm{dd}, J=3.0$ and $6.9 \mathrm{~Hz}, 2 \mathrm{H}),, 7.05-7.08(\mathrm{~m}, 4 \mathrm{H})$, 7.18-7.28 (m, 8H), $7.36(\mathrm{~d}, J=6.9 \mathrm{~Hz}, 2 \mathrm{H}), 7.81(\mathrm{~d}, J=7.5 \mathrm{~Hz}, 2 \mathrm{H})$. 


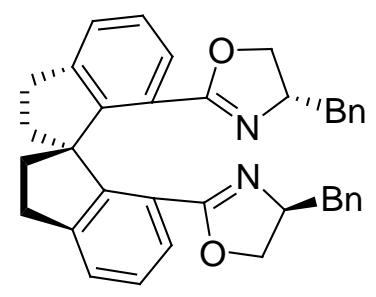

The compound $\left(S_{a}, S, S\right)$-1c was synthesized from $\left(S_{a}, S, S\right)-8 \mathbf{c}$ in $80 \%$ yield by the same procedure as that for $\left(S_{a}, S, S\right)-1 \mathbf{a}$. White solid, mp $49-52{ }^{\circ} \mathrm{C} ;[\alpha]^{20}{ }_{\mathrm{D}}=-220\left(\mathrm{c} 1.0, \mathrm{CHCl}_{3}\right) ;{ }^{1} \mathrm{H}$ NMR: $\delta 2.03(\mathrm{dd}, J=9.6$ and $4.4 \mathrm{~Hz}, 2 \mathrm{H}), 2.24(\mathrm{dd}, J=7.6$ and $4.4 \mathrm{~Hz}, 2 \mathrm{H}), 2.63-2.68(\mathrm{~m}$, 4H), 2.92-2.98 (m, 2H), 2.96-3.04 (m, 2H), 3.27 (t, $J=8.8 \mathrm{~Hz}, 2 \mathrm{H}), 3.57$ (t, $J=7.2 \mathrm{~Hz}, 2 \mathrm{H})$, 4.11-4.16 (m, 2H), 7.08-7.27 (m, 14H), 7.54-7.56 (d, $J=7.6 \mathrm{~Hz}, 2 \mathrm{H}) ;{ }^{13} \mathrm{C}$ NMR: $\delta 30.7$, $36.1,40.3,53.9,62.2,63.4,126.3,126.6,126.7,127.4,128.7,129.3,133.9,138.2,145.5$, 170.5; HRMS (EI) Calcd for $\mathrm{C}_{37} \mathrm{H}_{34} \mathrm{~N}_{2} \mathrm{O}_{2}$ : 538.2620; Found: 538.2614.

\section{Synthesis of $\left(S_{a}, S, S\right)-7,7^{\prime}$-bis(4-isopropyloxazolin-2-yl)-1,1'-spirobiindane $\left[\left(S_{a}, S, S\right)-1 \mathrm{c}\right]$}

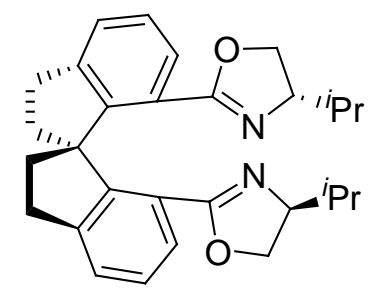

The compound $\left(S_{a}, S, S\right)$-1c was synthesized from $\left(S_{a}, S, S\right)-\mathbf{8 c}$ in $73 \%$ yield by the same procedure as that for $\left(S_{a}, S, S\right)-1 \mathbf{a}$. White solid, $\mathrm{mp}=64-66{ }^{\circ} \mathrm{C} ;[\alpha]^{20}{ }_{\mathrm{D}}=-353\left(\mathrm{c} 1.0, \mathrm{CHCl}_{3}\right)$; ${ }^{1} \mathrm{H}$ NMR: $\delta 0.67(\mathrm{~d}, J=6.9 \mathrm{~Hz}, 6 \mathrm{H}), 0.77(\mathrm{~d}, J=6.6 \mathrm{~Hz}, 6 \mathrm{H}), 1.11-1.20$ (m, 2H), $2.23(\mathrm{dd}, J$ $=7.2$ and $4.5 \mathrm{~Hz}, 2 \mathrm{H}), 2.62-2.72(\mathrm{~m}, 2 \mathrm{H}), 2.91-3.07(\mathrm{~m}, 6 \mathrm{H}), 3.33(\mathrm{dd}, J=7.8$ and $1.5 \mathrm{~Hz}$, 2H), 3.48-3.62 (m, 4H), 7.15 (t, J=7.5 Hz, 2H), 7.30 (d, J=7.2 Hz, 2H), 7.54 (d, J= $7.8 \mathrm{~Hz}$, $2 \mathrm{H}) ;{ }^{13} \mathrm{C}$ NMR: $\delta 17.8,19.6,30.6,32.4,38.4,69.6,72.3,125.8,126.4,127.8,145.1,148.5$, 163.4; HRMS (EI) Calcd for $\mathrm{C}_{29} \mathrm{H}_{34} \mathrm{~N}_{2} \mathrm{O}_{2}$ : 442.2620; Found: 442.2633. 


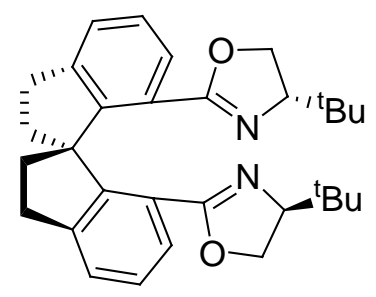

The compound $\left(S_{a}, S, S\right)$-1d was synthesized from $\left(S_{a}, S, S\right)-\mathbf{8 d}$ in $25 \%$ yield by the same procedure as that for $\left(S_{a}, S, S\right)-\mathbf{1 a}$. White solid, mp $149-152{ }^{\circ} \mathrm{C} ;[\alpha]^{20}=-395\left(c 1.0, \mathrm{CHCl}_{3}\right)$; ${ }^{1} \mathrm{H}$ NMR: $\delta 0.75(\mathrm{~s}, 18 \mathrm{H}), 2.19-2.25(\mathrm{~m}, 2 \mathrm{H}), 2.46-2.57(\mathrm{~m}, 2 \mathrm{H}), 2.91-3.07(\mathrm{~m}, 6 \mathrm{H})$, 3.58-3.64 (m, 4H), 7.16 (t, $J=7.5 \mathrm{~Hz}, 2 \mathrm{H}), 7.33$ (d, $J=7.5 \mathrm{~Hz}, 2 \mathrm{H}), 7.71(\mathrm{~d}, J=7.5 \mathrm{~Hz}, 2 \mathrm{H})$; ${ }^{13}$ C NMR: $\delta 25.8,30.6,33.7,38.7,68.1,74.9,124.2,125.9,126.5,128.7,144.9,149.0,164.1$; HRMS (EI) Calcd for $\mathrm{C}_{31} \mathrm{H}_{38} \mathrm{~N}_{2} \mathrm{O}_{2}$ : 470.2933; Found: 470.2922.

\section{General Procedure for Cu-Catalyzed Asymmetric Insertion of Carbenoid into} Anilines

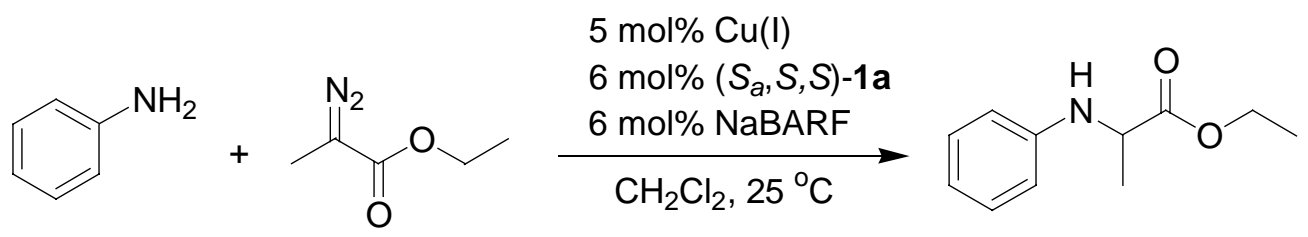

The $\mathrm{CuCl}(1.0 \mathrm{mg}, 0.01 \mathrm{mmol}, 5 \mathrm{~mol} \%),\left(S_{a}, S, S\right)-\mathbf{1 a}(6.1 \mathrm{mg}, 0.012 \mathrm{mmol}, 6 \mathrm{~mol} \%)$ and NaBARF. $\left(\mathrm{H}_{2} \mathrm{O}\right)_{1.5}(11.3 \mathrm{mg}, 0.012 \mathrm{mmol}, 6 \mathrm{~mol} \%)$ were introduced into an oven-dried Schlenk tube in argon-filled glovebox. $\mathrm{CH}_{2} \mathrm{Cl}_{2}(2 \mathrm{~mL})$ was injected and the solution was stirred at room temperature under the argon atmosphere for $2 \mathrm{~h}$, followed by additions of aniline (19 mg, $0.2 \mathrm{mmol}$ ) and ethyl $\alpha$-diazopropionate $(26 \mathrm{mg}, 0.2 \mathrm{mmol})$. The resulting mixture was stirred for $2 \mathrm{~h}$ and the product was purified by flash chromatography (ethyl acetate/petroleum ether $=1: 10$ ). 


\section{Analytical Data for N-H Insertion Products}

\section{(R)-(+)-Ethyl 2-(phenylamino)propionate (4a) ${ }^{4}$}<smiles>CCOC(=O)C(C)Nc1ccccc1</smiles>

Colorless oil, 94\% yield, ${ }^{1} \mathrm{H}$ NMR: $\delta 1.25$ (t, $\left.J=7.2 \mathrm{~Hz}, 3 \mathrm{H}\right), 1.47$ (d, $\left.J=6.4 \mathrm{~Hz}, 3 \mathrm{H}\right)$, 4.09-4.21 (m, 4H), $6.61(\mathrm{~d}, J=8.4 \mathrm{~Hz}, 2 \mathrm{H}), 6.74(\mathrm{t}, J=7.2 \mathrm{~Hz}, 1 \mathrm{H}), 7.17$ (t, $J=7.6 \mathrm{~Hz}, 2 \mathrm{H})$; 98\% ee [HPLC condition: Chiralpak AS column, $n-H e x a n e / 2-\mathrm{PrOH}=98: 2$, flow rate $=1.0$ $\mathrm{mL} / \mathrm{min}$, wavelength $=254 \mathrm{~nm}, t_{\mathrm{R}}=5.65 \mathrm{~min}$ for major isomer, $t_{\mathrm{R}}=6.26 \mathrm{~min}$ for minor isomer $] ; \alpha]^{20}{ }_{\mathrm{D}}=+98.8(\mathrm{c} 1.0, \mathrm{EtOH})$ and $[\alpha]^{20}{ }_{\mathrm{D}}=+58.7\left(c_{1} 1.0, \mathrm{CHCl}_{3}\right) .\left[\mathrm{lit}^{5}:[\alpha]_{\mathrm{D}}=+5.9(c\right.$ $\left.0.09, \mathrm{CHCl}_{3}\right)$ for $>75 \%$ ee, $\left.(R)\right]$.

\section{(+)-Ethyl 2-(p-toluidino)propionate (4b) ${ }^{4}$}<smiles>CCOC(=O)C(C)Nc1ccc(C)cc1</smiles>

Colorless oil, 94\% yield, ${ }^{1} \mathrm{H}$ NMR: $\delta 1.24$ (t, $\left.J=7.2 \mathrm{~Hz}, 3 \mathrm{H}\right), 1.44(\mathrm{~d}, J=6.8 \mathrm{~Hz}, 3 \mathrm{H}), 2.23$ (s, 3H), 4.01-4.20 (m, 4H), $6.54(\mathrm{~d}, J=8.4 \mathrm{~Hz}, 2 \mathrm{H}), 6.98(\mathrm{~d}, J=8.4 \mathrm{~Hz}, 2 \mathrm{H})$; 91\% ee [HPLC condition: Chiralpak AS column, $n$-Hexane $/ 2-\mathrm{PrOH}=98: 2$, flow rate $=1.0 \mathrm{~mL} / \mathrm{min}$, wavelength $=254 \mathrm{~nm}, t_{\mathrm{R}}=8.17 \min$ for major isomer, $t_{\mathrm{R}}=9.48 \min$ for minor isomer]; $[\alpha]^{20}{ }_{\mathrm{D}}=+59.8($ c $0.9, \mathrm{EtOH})$.

4 Bachmann, S.; Fielenbach, D.; Jøgensen, K. A. Org. Biomol. Chem. 2004, 2, 3044.

${ }^{5}$ Kurkin, A. V.; Nesterov, V. V.; Karchava, A. V.; Yurovskaya, M. A. Chem. Heterocycl. Compd. 2003, 39,1466 


\section{(+)-Ethyl 2-(p-methoxyphenylamino)propionate (4c) ${ }^{4}$}<smiles>CCOC(=O)C(C)Nc1ccc(OC)cc1</smiles>

Colorless oil, 96\% yield, ${ }^{1} \mathrm{H}$ NMR: $\delta 1.23$ (t, $\left.J=6.8 \mathrm{~Hz}, 3 \mathrm{H}\right), 1.44(\mathrm{~d}, J=6.8 \mathrm{~Hz}, 3 \mathrm{H}), 3.73$ (s, 3H), $3.87(\mathrm{~s}, 1 \mathrm{H}), 4.04(\mathrm{q}, J=6.8 \mathrm{~Hz}, 1 \mathrm{H}), 4.16(\mathrm{q}, J=7.2 \mathrm{~Hz}, 2 \mathrm{H}), 6.59$ (d, $J=8.8 \mathrm{~Hz}$, 2H), $6.76(\mathrm{~d}, J=8.8 \mathrm{~Hz}, 2 \mathrm{H}) ; 85 \%$ ee [HPLC condition: Chiralpak AS column, $n$-Hexane $/ 2-\mathrm{PrOH}=98: 2$, flow rate $=1.0 \mathrm{~mL} / \mathrm{min}$, wavelength $=254 \mathrm{~nm}, t_{\mathrm{R}}=21.75 \mathrm{~min}$ for minor isomer, $t_{\mathrm{R}}=23.36 \mathrm{~min}$ for major isomer $\left.] ; \alpha\right]^{20}{ }_{\mathrm{D}}=+41.8($ ( $1.0, \mathrm{EtOH})$.

(+)-Ethyl 2-(p-chlorophenylamino)propionate (4d)<smiles>CCOC(=O)C(C)Nc1ccc(Cl)cc1</smiles>

Colorless oil, 92\% yield, ${ }^{1} \mathrm{H}$ NMR: $\delta 1.24(\mathrm{t}, J=7.2 \mathrm{~Hz}, 3 \mathrm{H}), 1.43(\mathrm{~d}, J=6.8 \mathrm{~Hz}, 3 \mathrm{H}), 4.05$ $(\mathrm{q}, J=6.8 \mathrm{~Hz}, 1 \mathrm{H}), 4.17(\mathrm{q}, J=7.2 \mathrm{~Hz}, 2 \mathrm{H}), 6.50$ (d, $J=8.8 \mathrm{~Hz}, 2 \mathrm{H}), 7.09$ (d, $J=8.8 \mathrm{~Hz}$, $2 \mathrm{H}) ;{ }^{13} \mathrm{C}$ NMR: $\delta 14.4,18.9,52.3,61.4,114.7,123.0,129.3,145.5,174.5$; HRMS (EI) Calcd for $\mathrm{C}_{11} \mathrm{H}_{14} \mathrm{NO}_{2} \mathrm{Cl}$ : 227.0713, Found: 227.0716; 98\% ee [HPLC condition: Chiralpak AS column, $n$-Hexane $/ 2-\mathrm{PrOH}=98: 2$, flow rate $=1.0 \mathrm{~mL} / \mathrm{min}$, wavelength $=254 \mathrm{~nm}, t_{\mathrm{R}}=9.40$ $\min$ for minor isomer, $t_{\mathrm{R}}=10.05 \mathrm{~min}$ for major isomer $] ;[\alpha]^{20}{ }_{\mathrm{D}}=+101(c 1.0, \mathrm{EtOH})$.

(+)-Ethyl 2-(p-bromophenylamino)propionate (4e) 


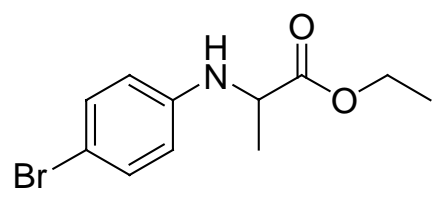

Colorless oil, 95\% yield, ${ }^{1} \mathrm{H}$ NMR: $\delta 1.24(\mathrm{t}, J=7.2 \mathrm{~Hz}, 3 \mathrm{H}), 1.44(\mathrm{~d}, J=6.8 \mathrm{~Hz}, 3 \mathrm{H})$, $4.02-4.09$ (m, 1H), 4.15-4.24 (m, 3H), $6.46(\mathrm{~d}, J=9.2 \mathrm{~Hz}, 2 \mathrm{H}), 7.23(\mathrm{~d}, J=9.2 \mathrm{~Hz}, 2 \mathrm{H}) ;{ }^{13} \mathrm{C}$ NMR: $\delta 14.4,18.9,52.2,61.5,110.1,115.2,132.2,145.9$, 174.4; HRMS (EI) Calcd for $\mathrm{C}_{11} \mathrm{H}_{14} \mathrm{NO}_{2} \mathrm{Br}$ : 271.0208, Found: 271.0211; 98\% ee [HPLC condition: Chiralpak AS column, $n$-Hexane $/ 2-\mathrm{PrOH}=98: 2$, flow rate $=1.0 \mathrm{~mL} / \mathrm{min}$, wavelength $=254 \mathrm{~nm}, t_{\mathrm{R}}=10.13 \mathrm{~min}$ for minor isomer, $t_{\mathrm{R}}=12.21 \mathrm{~min}$ for major isomer $] ;[\alpha]^{20}{ }_{\mathrm{D}}=+78.9($ c 1.0, EtOH $)$.

\section{(+)-Ethyl 2-(m-toluidino)propionate (4f)}

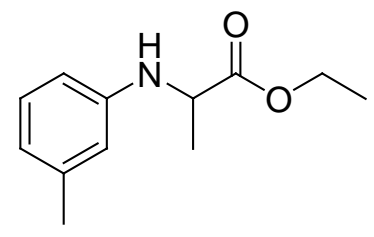

Colorless oil, 92\% yield, ${ }^{1} \mathrm{H}$ NMR: $\delta 1.25$ (t, $\left.J=7.2 \mathrm{~Hz}, 3 \mathrm{H}\right), 1.46(\mathrm{~d}, J=6.4 \mathrm{~Hz}, 3 \mathrm{H}), 2.26$ (s, 3H), 4.10-4.14 (m, 2H), 4.15-4.21 (m, 2H), 6.41-6.43 (m, 2H), $6.56(\mathrm{~d}, J=7.6 \mathrm{~Hz}, 1 \mathrm{H})$, 7.05 (t, $J=7.6 \mathrm{~Hz}, 1 \mathrm{H}) ;{ }^{13} \mathrm{C} \mathrm{NMR}: \delta 14.4,19.2,21.7,52.3,61.2,110.7,114.5,119.4,129.3$, 139.3, 146.9, 174.8; HRMS (EI) Calcd for $\mathrm{C}_{12} \mathrm{H}_{17} \mathrm{NO}_{2}$ : 207.1259; Found: 207.1263; 96\% ee [HPLC condition: Chiralpak AS column, $n$-Hexane $/ 2-\mathrm{PrOH}=98: 2$, flow rate $=1.0 \mathrm{~mL} / \mathrm{min}$, wavelength $=254 \mathrm{~nm}, t_{\mathrm{R}}=6.10 \mathrm{~min}$ for major isomer, $t_{\mathrm{R}}=6.60 \mathrm{~min}$ for minor isomer]; $[\alpha]^{20}=+72.2(c 0.8, \mathrm{EtOH})$.

(+)-Ethyl 2-(m-chlorophenylamino)propionate (4g) ${ }^{6}$

\footnotetext{
${ }^{6}$ Marilena, G.; Nicola, C.; Rita, L. M.; Maurizio, M.; Massimo, M. J. Med. Chem. 1999, 42, 336.
} 


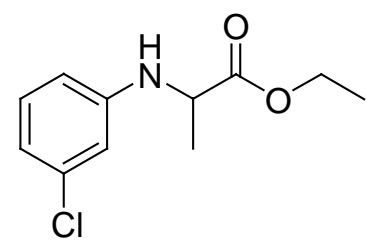

Colorless oil, 95\% yield, ${ }^{1} \mathrm{H}$ NMR: $\delta 1.26(\mathrm{t}, J=7.2 \mathrm{~Hz}, 3 \mathrm{H}), 1.46(\mathrm{~d}, J=6.8 \mathrm{~Hz}, 3 \mathrm{H})$, 4.07-4.27 (m, 4H), $6.46(\mathrm{dd}, J=8.4$ and $2.4 \mathrm{~Hz}, 1 \mathrm{H}), 6.56-6.57(\mathrm{~m}, 1 \mathrm{H}), 6.68-6.70(\mathrm{~m}, 1 \mathrm{H})$, $7.6(\mathrm{t}, J=8.0 \mathrm{~Hz}, 1 \mathrm{H}) ; 97 \%$ ee [HPLC condition: Chiralpak AS column, $n-\mathrm{Hexane} / 2-\mathrm{PrOH}=$ $98: 2$, flow rate $=1.0 \mathrm{~mL} / \mathrm{min}$, wavelength $=254 \mathrm{~nm}, t_{\mathrm{R}}=6.59 \mathrm{~min}$ for major isomer, $t_{\mathrm{R}}=$ $8.27 \mathrm{~min}$ for minor isomer]; $[\alpha]^{20}{ }_{\mathrm{D}}=+82.6$ (c 1.2, EtOH).

(+)-Ethyl 2-(m-bromophenylamino)propionate (4h)

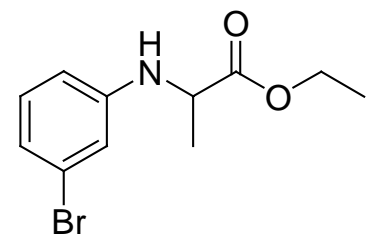

Colorless oil, 96\% yield, ${ }^{1} \mathrm{H}$ NMR: $\delta 1.19(\mathrm{t}, J=6.9 \mathrm{~Hz}, 3 \mathrm{H}), 1.38(\mathrm{~d}, J=6.9 \mathrm{~Hz}, 3 \mathrm{H})$, 3.96-4.06 (m, 1H), 4.10-4.18 (m, 3H), 6.42-6.45 (m, 1H), 6.66-6.67 (m, 1H), 6.75-6.78 (m, 1H), $6.93(\mathrm{t}, J=8.1 \mathrm{~Hz}, 1 \mathrm{H}) ;{ }^{13} \mathrm{C} \mathrm{NMR}: \delta 14.2,18.7,51.8,61.3,112.1,116,0,121.0,123.3$, 130.5, 147.9, 174.1; HRMS (EI) Calcd for $\mathrm{C}_{11} \mathrm{H}_{14} \mathrm{NO}_{2} \mathrm{Br}$ : 271.0208. Found: 271.0206; 98\% ee [HPLC condition: Chiralpak AS column, $n$-Hexane/2-PrOH $=98: 2$, flow rate $=1.0$ $\mathrm{mL} / \mathrm{min}$, wavelength $=254 \mathrm{~nm}, t_{\mathrm{R}}=7.11 \mathrm{~min}$ for major isomer, $t_{\mathrm{R}}=8.54 \mathrm{~min}$ for minor isomer $] ; \alpha]^{20}{ }_{\mathrm{D}}=+71.7$ (c 1.0, EtOH).

\section{(+)-Ethyl 2-(o-toluidino)propionate (4i)}<smiles>CCOC(=O)C(C)Nc1ccccc1C</smiles> 
Colorless oil, 95\% yield, ${ }^{1} \mathrm{H}$ NMR: $\delta 1.26(\mathrm{t}, J=7.2 \mathrm{~Hz}, 3 \mathrm{H}), 1.51(\mathrm{~d}, J=6.8 \mathrm{~Hz}, 3 \mathrm{H}), 2.21$ (s, 3H), 4.07-4.09 (m, 1H), 4.16-4.23 (m, 3H), 6.54 (d, J=8.0 Hz, 1H), 6.67-6.71 (m, 1H), 7.06-7.11 (m, 2H); ${ }^{13} \mathrm{C}$ NMR: $\delta 14.4,17.6,19.3,52.2,61.3,110.6,118.0,122.8,127.3,130.6$, 144.9, 174.9; HRMS (EI) Calcd for $\mathrm{C}_{12} \mathrm{H}_{17} \mathrm{NO}_{2}$ : 207.1259; Found: 207.1254; 98\% ee [HPLC condition: Chiralpak AD-H column, $n$-Hexane $/ 2-\mathrm{PrOH}=99: 1$, flow rate $=1.0 \mathrm{~mL} / \mathrm{min}$, wavelength $=254 \mathrm{~nm}, t_{\mathrm{R}}=13.01 \mathrm{~min}$ for major isomer, $t_{\mathrm{R}}=15.71 \mathrm{~min}$ for minor isomer]; $[\alpha]^{20}{ }_{\mathrm{D}}=+31.9(c$ 1.0, EtOH $)$.

(+)-Ethyl 2-(o-methoxyphenylamino)propionate $(4 \mathrm{j}){ }^{4}$<smiles>CCOC(=O)C(C)Nc1ccccc1OC</smiles>

Colorless oil, 86\% yield, ${ }^{1} \mathrm{H}$ NMR: $\delta 1.24(\mathrm{t}, J=7.0 \mathrm{~Hz}, 3 \mathrm{H}), 1.50(\mathrm{~d}, J=6.4 \mathrm{~Hz}, 3 \mathrm{H}), 3.85$ (s, 3H), 4.11-4.21 (m, 3H), $4.70(\mathrm{~d}, J=7.2 \mathrm{~Hz}, 1 \mathrm{H}), 6.53(\mathrm{~d}, J=7.6 \mathrm{~Hz}, 1 \mathrm{H}), 6.68-6.71(\mathrm{~m}$, 1H), 6.77-6.84 (m, 2H); 98\% ee [HPLC condition: Chiralpak AD-H column, $n$-Hexane $/ 2-\mathrm{PrOH}=99: 1$, flow rate $=1.0 \mathrm{~mL} / \mathrm{min}$, wavelength $=254 \mathrm{~nm}, t_{\mathrm{R}}=21.56 \mathrm{~min}$ for major isomer, $t_{\mathrm{R}}=25.66 \mathrm{~min}$ for minor isomer $] ;[\alpha]^{20}{ }_{\mathrm{D}}=+23.7($ c 1.1, EtOH).

\section{(+)-Ethyl 2-(o-chlorophenylamino)propanoate (4k)}

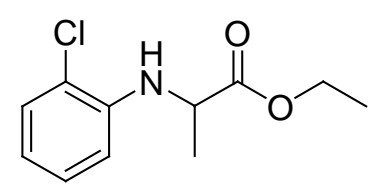

Colorless oil, 95\% yield, ${ }^{1} \mathrm{H}$ NMR: $\delta 1.25$ (t, $\left.J=7.2 \mathrm{~Hz}, 3 \mathrm{H}\right), 1.52(\mathrm{~d}, J=7.2 \mathrm{~Hz}, 3 \mathrm{H})$, 4.13-4.22 (m, 3H), $4.80(\mathrm{~d}, J=7.6 \mathrm{~Hz}, 1 \mathrm{H}), 6.57(\mathrm{~d}, J=8.4 \mathrm{~Hz}, 1 \mathrm{H}), 6.64-6.68(\mathrm{~m}, 1 \mathrm{H})$, 7.09-7.13 (m, 1H), 7.25-7.27(m, 1H); ${ }^{13} \mathrm{C}$ NMR $\delta 14.37,19.01,52.08,61.48,111.83,118.31$, 
119.91, 127.95, 129.61, 142.85, 174.10; HRMS (EI) Calcd for $\mathrm{C}_{11} \mathrm{H}_{14} \mathrm{NO}_{2} \mathrm{Cl}$ : 227.0713, Found: $227.0710 ; 88 \%$ ee [HPLC condition: Chiralcel OD-H column, $n$-Hexane/2-PrOH = $98: 2$, flow rate $=1.0 \mathrm{~mL} / \mathrm{min}$, wavelength $=254 \mathrm{~nm}, t_{\mathrm{R}}=7.00 \mathrm{~min}$ for major isomer, $t_{\mathrm{R}}=$ 22.99 min for minor isomer $] ; \alpha]^{20}{ }_{D}=+9.8$ (c 1.1, EtOH).

\section{(-)-Ethyl 2-(naphthalen-1-ylamino)propionate (4l) ${ }^{4}$}

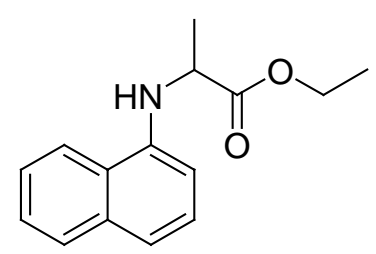

Colorless oil, 89\% yield, ${ }^{1} \mathrm{H}$ NMR: $\delta 1.28(\mathrm{t}, J=7.2 \mathrm{~Hz}, 3 \mathrm{H}), 1.61(\mathrm{~d}, J=6.8 \mathrm{~Hz}, 3 \mathrm{H})$, 4.20-4.34 (m, 3H), $4.96(\mathrm{~d}, J=4.0 \mathrm{~Hz}, 1 \mathrm{H}), 6.54$ (d, $J=7.2 \mathrm{~Hz}, 1 \mathrm{H}), 7.26-7.34(\mathrm{~m}, 2 \mathrm{H})$, 7.45-7.47 (m, 2H), 7.78-7.80 (m, 1H), 7.90-7.92 (m, 1H); 98\% ee [HPLC condition: Chiralpak AD-H column, $n-\mathrm{Hexane} / 2-\mathrm{PrOH}=95: 5$, flow rate $=1.0 \mathrm{~mL} / \mathrm{min}$, wavelength $=$ $254 \mathrm{~nm}, t_{\mathrm{R}}=7.81 \mathrm{~min}$ for major isomer, $t_{\mathrm{R}}=10.18 \min$ for minor isomer $] ;[\alpha]^{20}{ }_{\mathrm{D}}=-23.9(\mathrm{c}$ $1.1, \mathrm{EtOH})$.

(+)-Ethyl 2-(naphthalen-2-ylamino)propionate (4m)<smiles>CCOC(=O)C(C)Nc1ccc2ccccc2c1</smiles>

White solid, 91\% yield, $\mathrm{mp}=80-82{ }^{\circ} \mathrm{C} ;{ }^{1} \mathrm{H}$ NMR: $\delta 1.27(\mathrm{t}, J=7.2 \mathrm{~Hz}, 3 \mathrm{H}), 1.54(\mathrm{~d}, J=6.8$ $\mathrm{Hz}, 3 \mathrm{H}), 4.17-4.36(\mathrm{~m}, 4 \mathrm{H}), 6.78(\mathrm{~s}, 1 \mathrm{H}), 6.90-6.93(\mathrm{~m}, 1 \mathrm{H}), 7.21$ (t, J=6.8 Hz, 1H), 7.36 (t, $J=8.0 \mathrm{~Hz}, 1 \mathrm{H}), 7.59-7.68(\mathrm{~m}, 3 \mathrm{H}) ;{ }^{13} \mathrm{C}$ NMR: $\delta 14.2,18.8,52.1,61.2,105.4,118.1,122.4$, 126.1, 126.3, 127.6, 129.1, 135.0, 144.3, 174.5; HRMS (EI) Calcd for $\mathrm{C}_{15} \mathrm{H}_{17} \mathrm{NO}_{2}$ : 243.1259. Found: 243.1266; 98\% ee [HPLC condition: Chiralpak AS column, $n-H e x a n e / 2-\mathrm{PrOH}=98: 2$, flow rate $=1.0 \mathrm{~mL} / \mathrm{min}$, wavelength $=254 \mathrm{~nm}, t_{\mathrm{R}}=9.20 \mathrm{~min}$ for major isomer, $t_{\mathrm{R}}=11.26$ 
$\min$ for minor isomer $] ;[\alpha]^{20}{ }_{\mathrm{D}}=+173(\mathrm{c} 1.0, \mathrm{EtOH})$.

\section{(+)-Methyl 2-(phenylamino)propionate (4n) ${ }^{7}$}<smiles>COC(=O)C(C)Nc1ccccc1</smiles>

Colorless oil, 78\% yield, ${ }^{1} \mathrm{H}$ NMR: $\delta 1.48$ (d, $\left.J=5.6 \mathrm{~Hz}, 3 \mathrm{H}\right), 3.73$ (s, 3H), 4.15 (brs, 2H), $6.61(\mathrm{~d}, J=8.4 \mathrm{~Hz}, 2 \mathrm{H}), 6.75(\mathrm{t}, J=7.2 \mathrm{~Hz}, 1 \mathrm{H}), 7.18(\mathrm{t}, J=8.0 \mathrm{~Hz}, 2 \mathrm{H}) ; 96 \%$ ee [HPLC condition: Chiralpak AS column, $n$-Hexane $/ 2-\mathrm{PrOH}=98: 2$, flow rate $=1.0 \mathrm{~mL} / \mathrm{min}$, wavelength $=254 \mathrm{~nm}, t_{\mathrm{R}}=9.79 \min$ for major isomer, $t_{\mathrm{R}}=10.94 \min$ for minor isomer]; $[\alpha]^{20}{ }_{\mathrm{D}}=+111(\mathrm{c} 1.0, \mathrm{EtOH})$.

(+)-tert-Butyl 2-(phenylamino)propionate (40) ${ }^{4}$

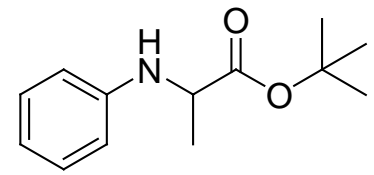

Colorless oil, 93\% yield, ${ }^{1} \mathrm{H}$ NMR: $\delta 1.43$ (s, 9H), 4.01 (q, $\left.J=6.8 \mathrm{~Hz}, 1 \mathrm{H}\right), 4.14$ (brs, 1H), $6.60(\mathrm{~d}, J=8.0 \mathrm{~Hz}, 2 \mathrm{H}), 6.72(\mathrm{t}, J=7.2 \mathrm{~Hz}, 1 \mathrm{H}), 7.16(\mathrm{t}, J=8.0 \mathrm{~Hz}, 2 \mathrm{H}) ; 96 \%$ ee [HPLC condition: Chiralpak AS column, $n$-Hexane $/ 2-\mathrm{PrOH}=98: 2$, flow rate $=1.0 \mathrm{~mL} / \mathrm{min}$, wavelength $=254 \mathrm{~nm}, t_{\mathrm{R}}=6.51 \mathrm{~min}$ for major isomer, $t_{\mathrm{R}}=8.60 \mathrm{~min}$ for minor isomer]; $[\alpha]^{20}=+43.3($ ( $1.0, \mathrm{EtOH})$.

(+)-Ethyl 2-(phenylamino)butyrate (4p)

\footnotetext{
7 Gately, D. A.; Norton, J. R. J. Am. Chem. Soc. 1996, 118, 3479
} 


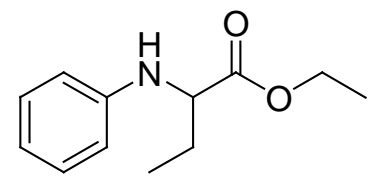

Colorless oil, 51\% yield, ${ }^{1} \mathrm{H}$ NMR: $\delta 1.00(\mathrm{t}, J=7.6 \mathrm{~Hz}, 3 \mathrm{H}), 1.25$ (t, $\left.J=7.2 \mathrm{~Hz}, 3 \mathrm{H}\right)$, $1.77-1.93(\mathrm{~m}, 2 \mathrm{H}), 4.02(\mathrm{t}, J=6.4 \mathrm{~Hz}, 2 \mathrm{H}), 4.19$ (q, $J=7.2 \mathrm{~Hz}, 2 \mathrm{H}), 6.62(\mathrm{~d}, J=7.6 \mathrm{~Hz}, 2 \mathrm{H})$, $6.72(\mathrm{t}, J=7.6 \mathrm{~Hz}, 1 \mathrm{H}), 7.17(\mathrm{t}, J=8.0 \mathrm{~Hz}, 2 \mathrm{H}) ;{ }^{13} \mathrm{C} \mathrm{NMR}: \delta 10.1,14.5,26.3,58.0,61.2$, 113.6, 118.4, 129.5, 147.1, 174.2; HRMS (EI) Calcd for $\mathrm{C}_{12} \mathrm{H}_{17} \mathrm{NO}_{2}$ : 207.1259; Found: 207.1266; 94\% ee [HPLC condition: Chiralpak AS column, n-Hexane/2-PrOH = 98:2, flow rate $=1.0 \mathrm{~mL} / \mathrm{min}$, wavelength $=254 \mathrm{~nm}, t_{\mathrm{R}}=6.41 \mathrm{~min}$ for major isomer, $t_{\mathrm{R}}=7.31 \mathrm{~min}$ for minor isomer $] ; \alpha]^{20}{ }_{\mathrm{D}}=+100(c 1.0, \mathrm{EtOH})$.

\section{(+)-Methyl 2-phenyl-2-(phenylamino)acetate (4q) ${ }^{4}$}<smiles>COC(=O)C(Nc1ccccc1)c1ccccc1</smiles>

White solid, 85\% yield, mp $=76-78{ }^{\circ} \mathrm{C} ;{ }^{1} \mathrm{H}$ NMR: $\delta 3.73(\mathrm{~s}, 3 \mathrm{H}), 4.96(\mathrm{~s}, 1 \mathrm{H}), 5.01(\mathrm{~s}, 1 \mathrm{H})$, $6.55(\mathrm{~d}, J=8.0 \mathrm{~Hz}, 2 \mathrm{H}), 6.69(\mathrm{t}, J=7.2 \mathrm{~Hz}, 1 \mathrm{H}), 7.12(\mathrm{t}, J=8.0 \mathrm{~Hz}, 2 \mathrm{H}), 7.30-7.37(\mathrm{~m}, 3 \mathrm{H})$, $7.49(\mathrm{~d}, J=7.2 \mathrm{~Hz}, 2 \mathrm{H}) ; 8 \%$ ee [HPLC condition: Chiralpak AS column, n-Hexane/2-PrOH = $98: 2$, flow rate $=1.0 \mathrm{~mL} / \mathrm{min}$, wavelength $=254 \mathrm{~nm}, t_{\mathrm{R}}=8.68 \mathrm{~min}$ for major isomer, $t_{\mathrm{R}}=$ 10.29 min for minor isomer $] ; \alpha]^{20}{ }_{\mathrm{D}}=-7.3(\mathrm{c} 1.0, \mathrm{EtOH})$.

\section{Ethyl 2-(methyl(phenyl)amino)propionate (4r) ${ }^{4}$}<smiles>CCOC(=O)C(C)N(C)c1ccccc1</smiles> 
Colorless oil, 93\% yield, ${ }^{1} \mathrm{H}$ NMR: $\delta 1.22$ (t, $\left.J=6.9 \mathrm{~Hz}, 3 \mathrm{H}\right), 1.47$ (d, $\left.J=7.2 \mathrm{~Hz}, 3 \mathrm{H}\right), 2.89$ (s, 3H), 4.14-4.17 (m, 2H), 4.47-4.54 (m, 1H), 6.75-6.80 (m, 3H), 7.21-7.26 (m, 2H); rac. [HPLC condition: Chiralpak AD-H column, $n$-Hexane/2-PrOH $=98: 2$, flow rate $=1.0$ $\mathrm{mL} / \mathrm{min}$, wavelength $\left.=254 \mathrm{~nm}, t_{\mathrm{R}}=6.67 \mathrm{~min}, t_{\mathrm{R}}=7.04 \mathrm{~min}\right]$.

\section{Ethyl 2-benzamidopropionate (4s) ${ }^{8}$}<smiles>CCOC(=O)C(C)NC(=O)c1ccccc1</smiles>

White solid, 55\% yield, $\mathrm{mp}=74-76{ }^{\circ} \mathrm{C} ;{ }^{1} \mathrm{H}$ NMR: $\delta 1.31(\mathrm{t}, J=7.2 \mathrm{~Hz}, 3 \mathrm{H}), 1.52(\mathrm{~d}, J=7.2$ $\mathrm{Hz}, 3 \mathrm{H}), 4.21-4.27(\mathrm{~m}, 2 \mathrm{H}), 4.74-4.79(\mathrm{~m}, 1 \mathrm{H}), 6.76(\mathrm{~m}, 1 \mathrm{H}), 7.42-7.45$ (m, 2H), 7.51-7.56 $(\mathrm{m}, 3 \mathrm{H})$; rac. [HPLC condition: Chiralcel OB column, $n$-Hexane/2-PrOH $=90: 10$, flow rate $=$ $1.0 \mathrm{~mL} / \mathrm{min}$, wavelength $\left.=235 \mathrm{~nm}, t_{\mathrm{R}}=21.22 \mathrm{~min}, t_{\mathrm{R}}=27.98 \mathrm{~min}\right]$.

8 Benati, L.; Nanni, D.; Sangiorgi, C.; Spagnolo, P. J. Org. Chem 1996, 64, 7836. 
4. NMR Spectra for New Ligands and Intermediates

(S)-1,1'-Spirobiindanyl-7,7'-dicarboxylic acid dimethyl ester

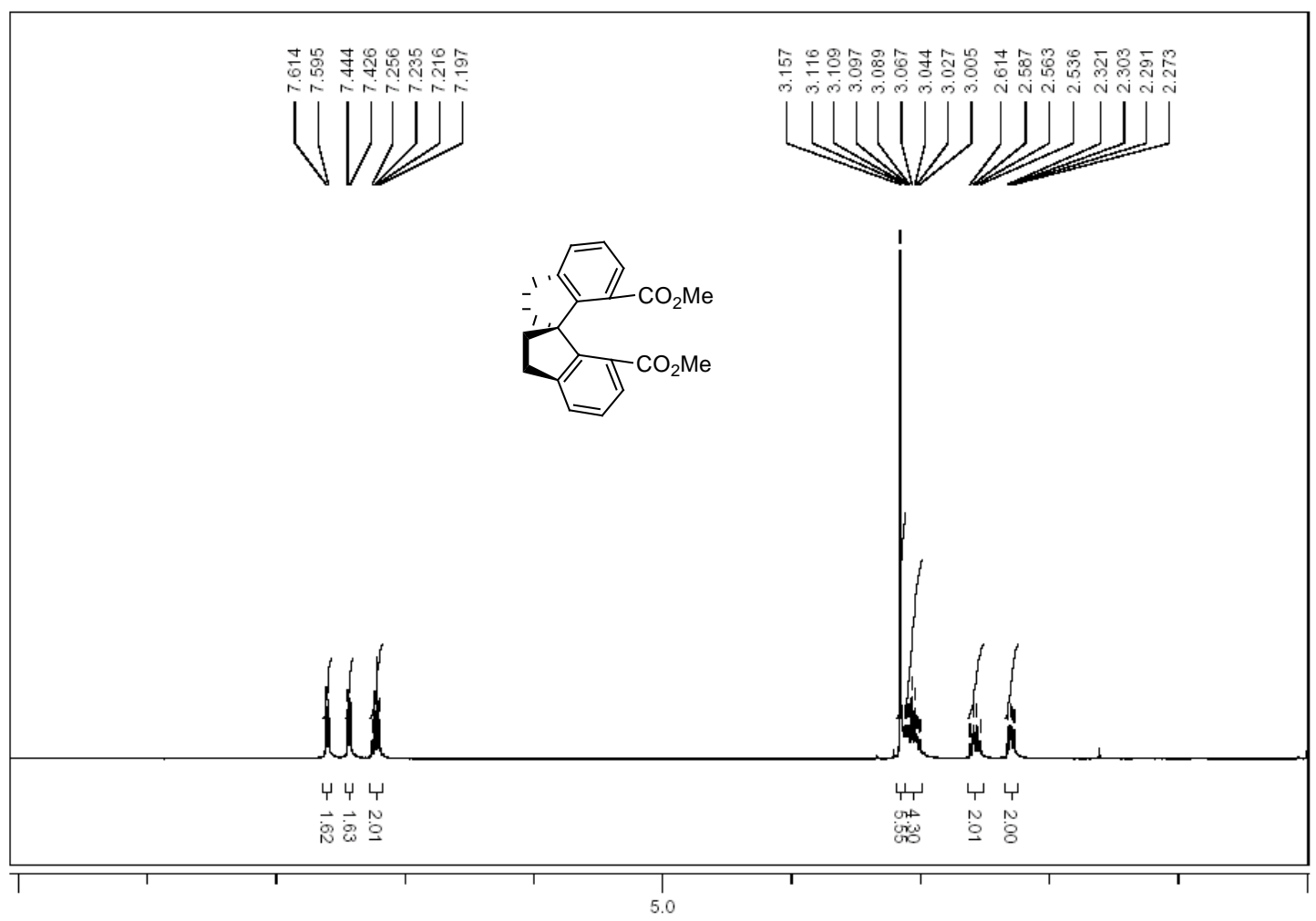

ppm (f1)

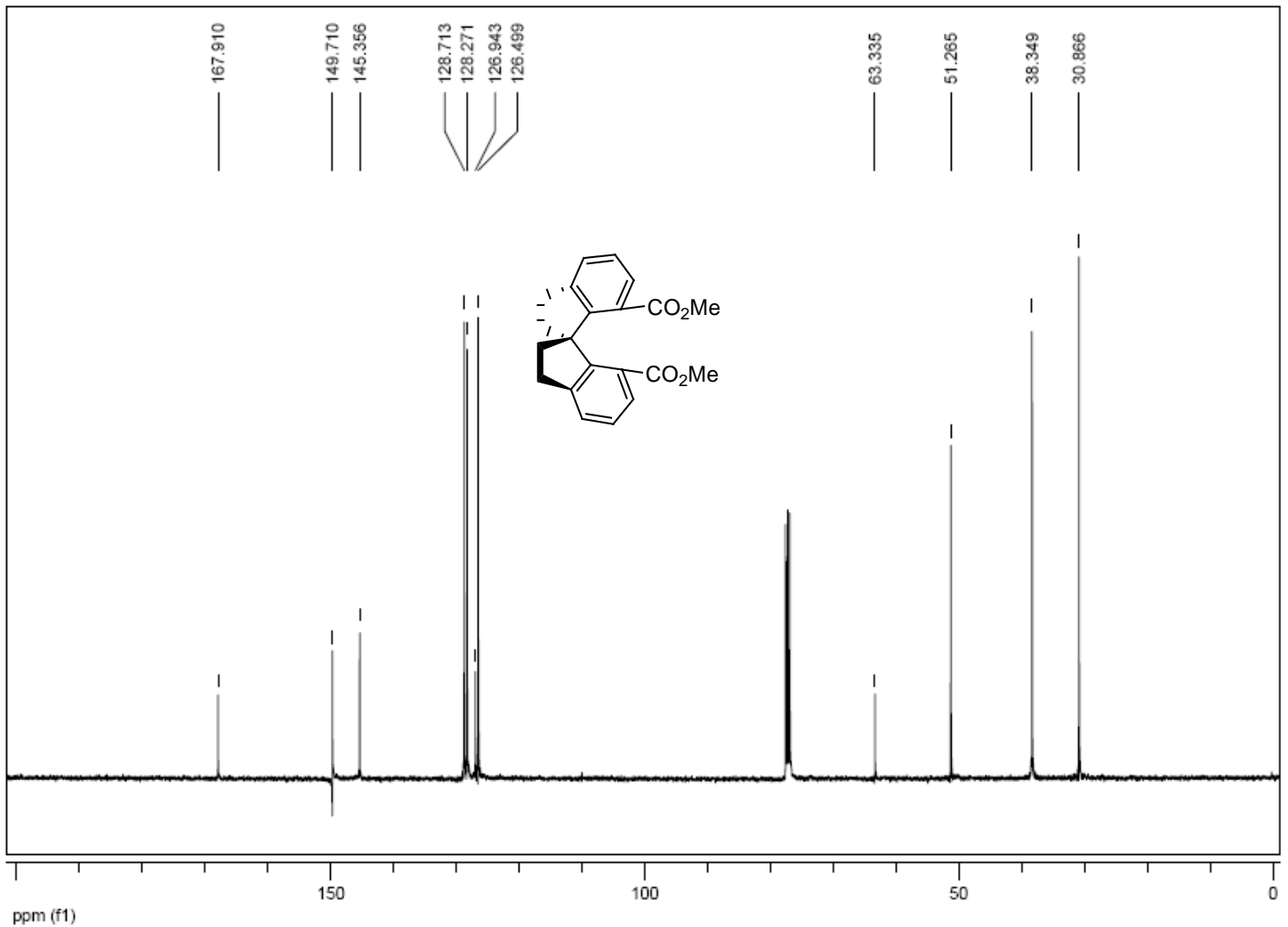


$\left(S_{a}, S, S\right)-7,7^{\prime}$-Bis[N-(1-benzyl-2-hydroxyethyl)amide]-1,1'-spirobiindane
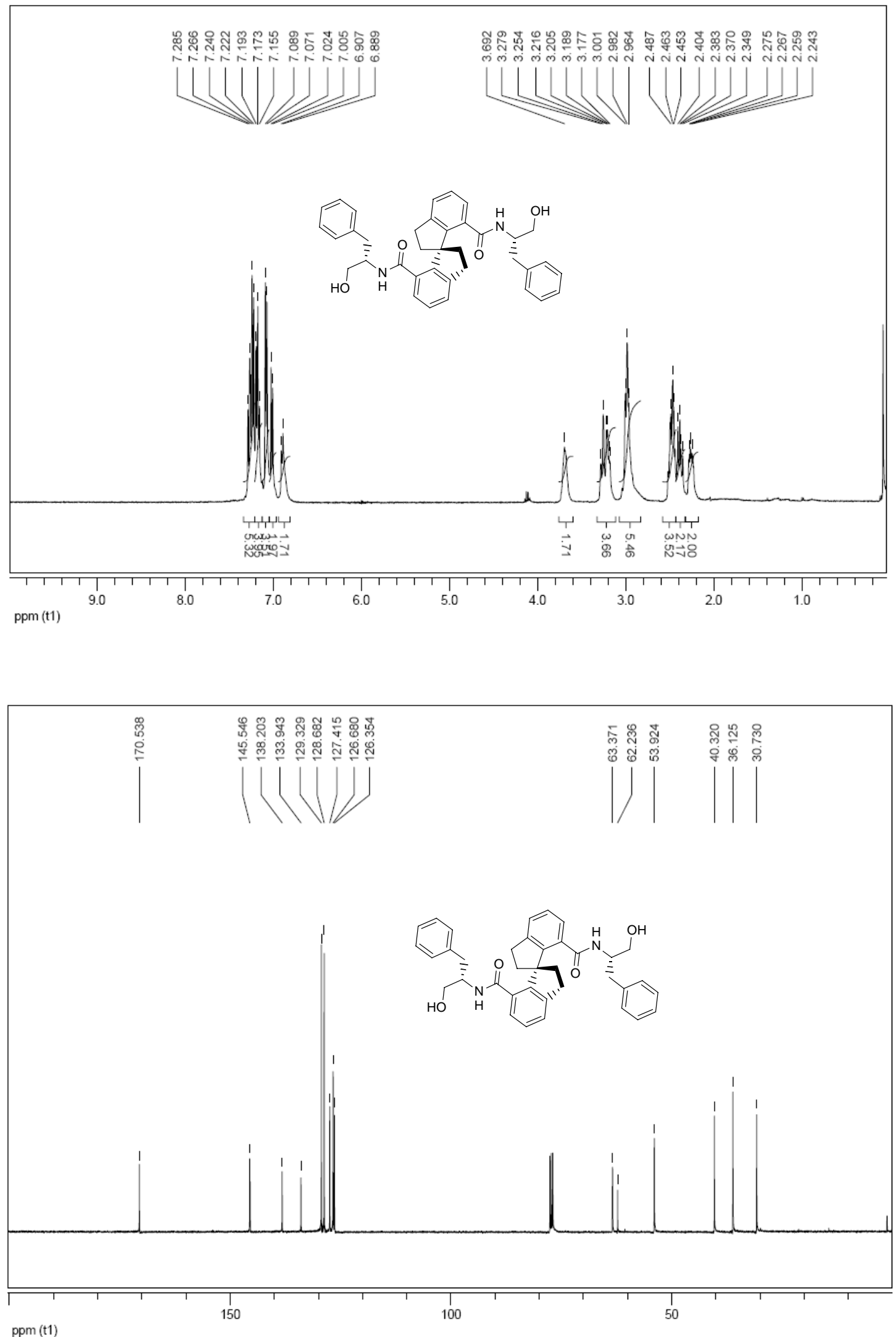
$\left(S_{a}, S, S\right)-7,7^{\prime}$-Bis[N-(1-hydroxymethyl-2-methylpropyl)amide]-1,1'-spirobiindane
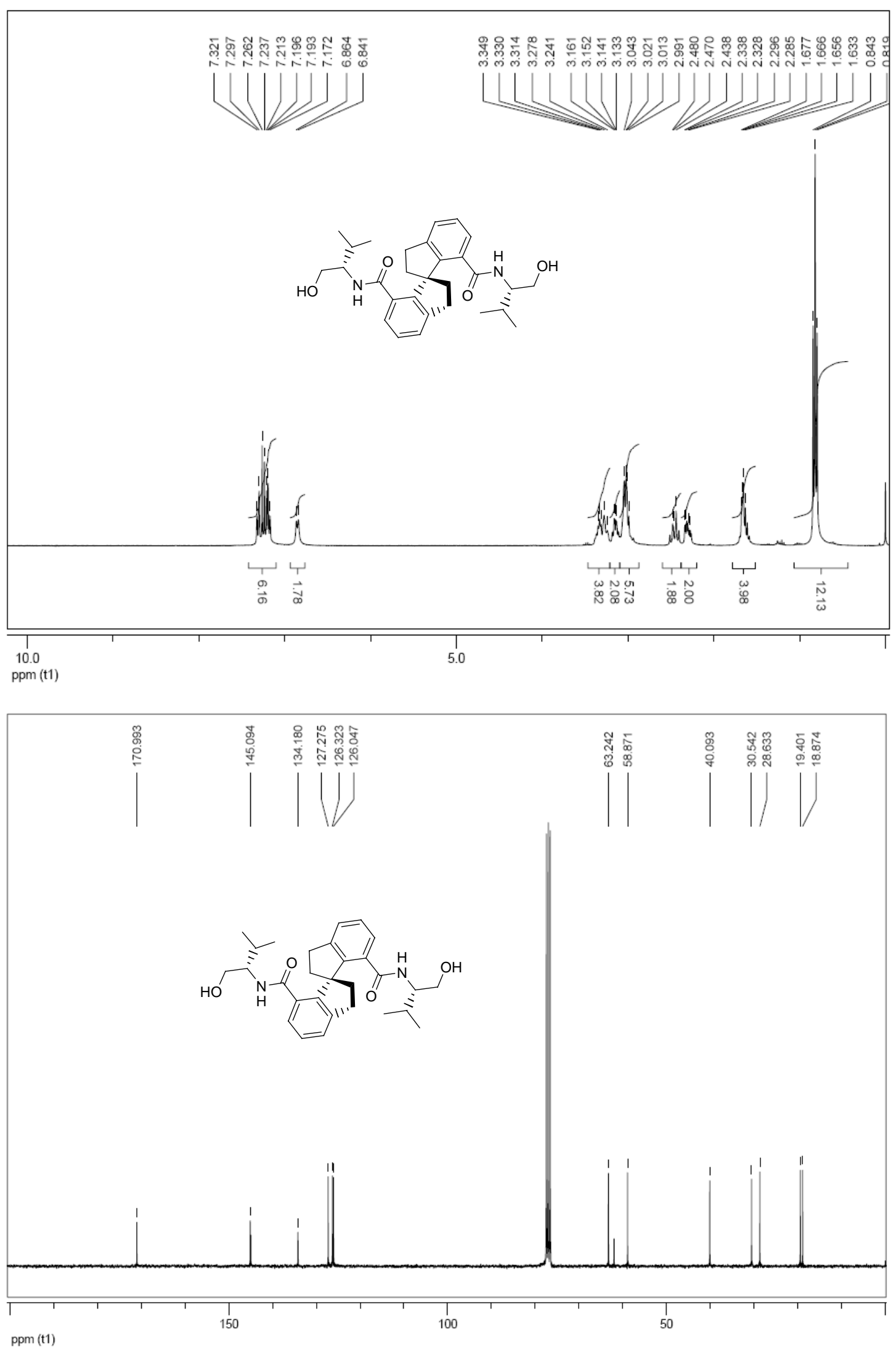
$\left(S_{a}, S, S\right)-7,7^{\prime}$-Bis[N-(2-hydroxy-1-tert-butylethyl)amide]-1,1'-spirobiindane
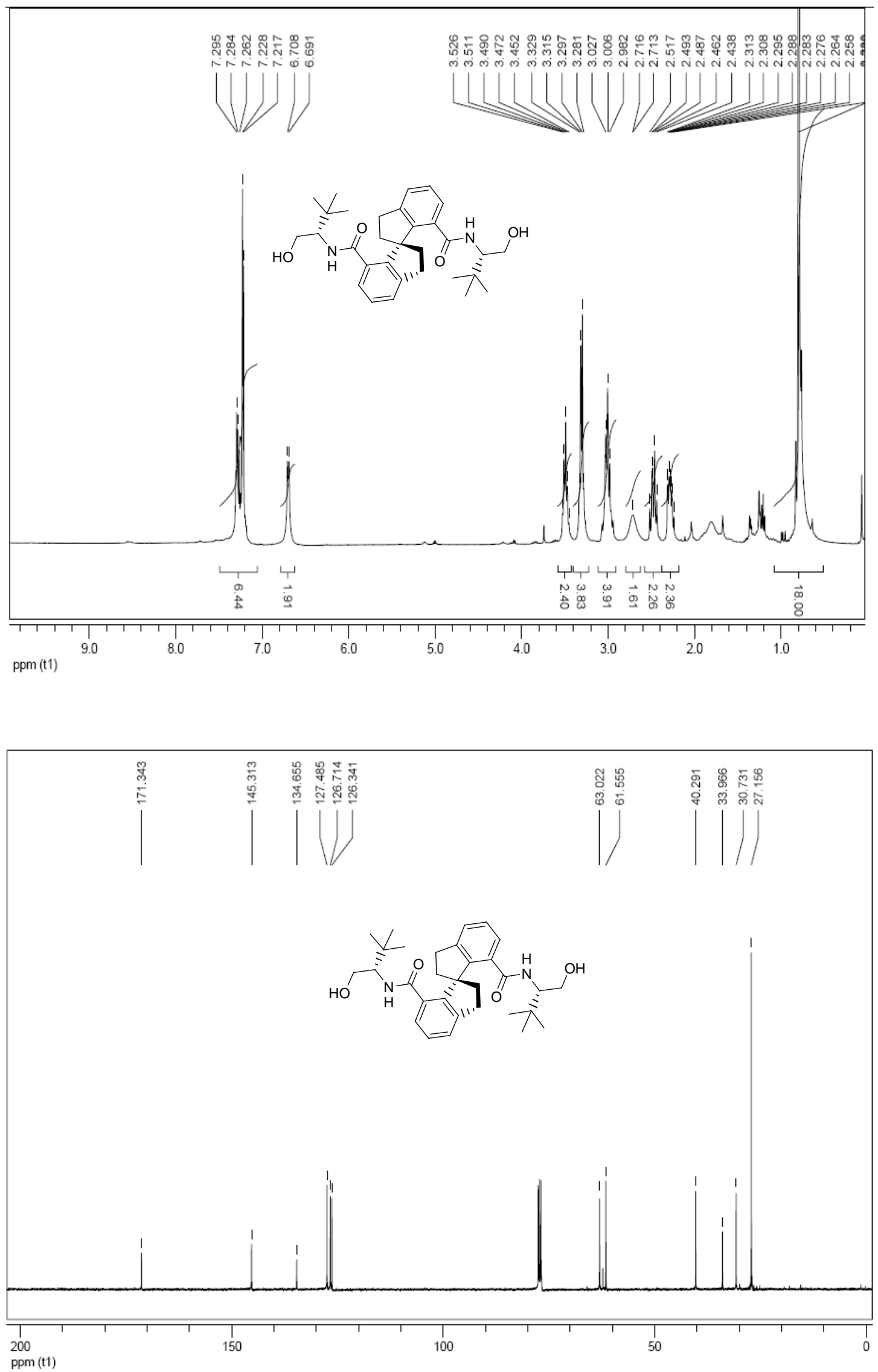


\section{$\left(S_{a}, S, S\right)-7,7^{\prime}$-Bis(4-benzyloxazolin-2-yl)-1,1'-spirobiindane}

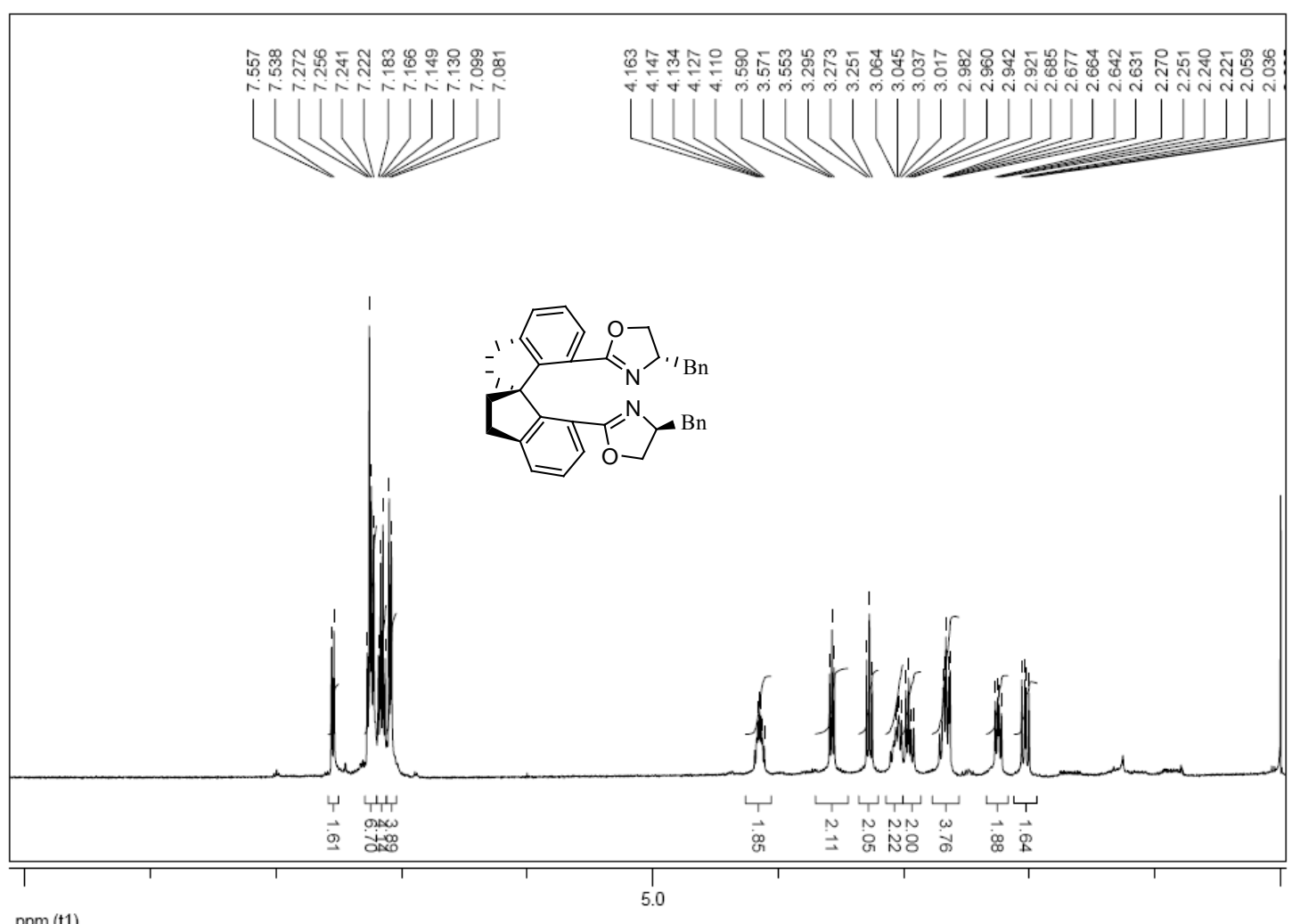

nnm (t1)

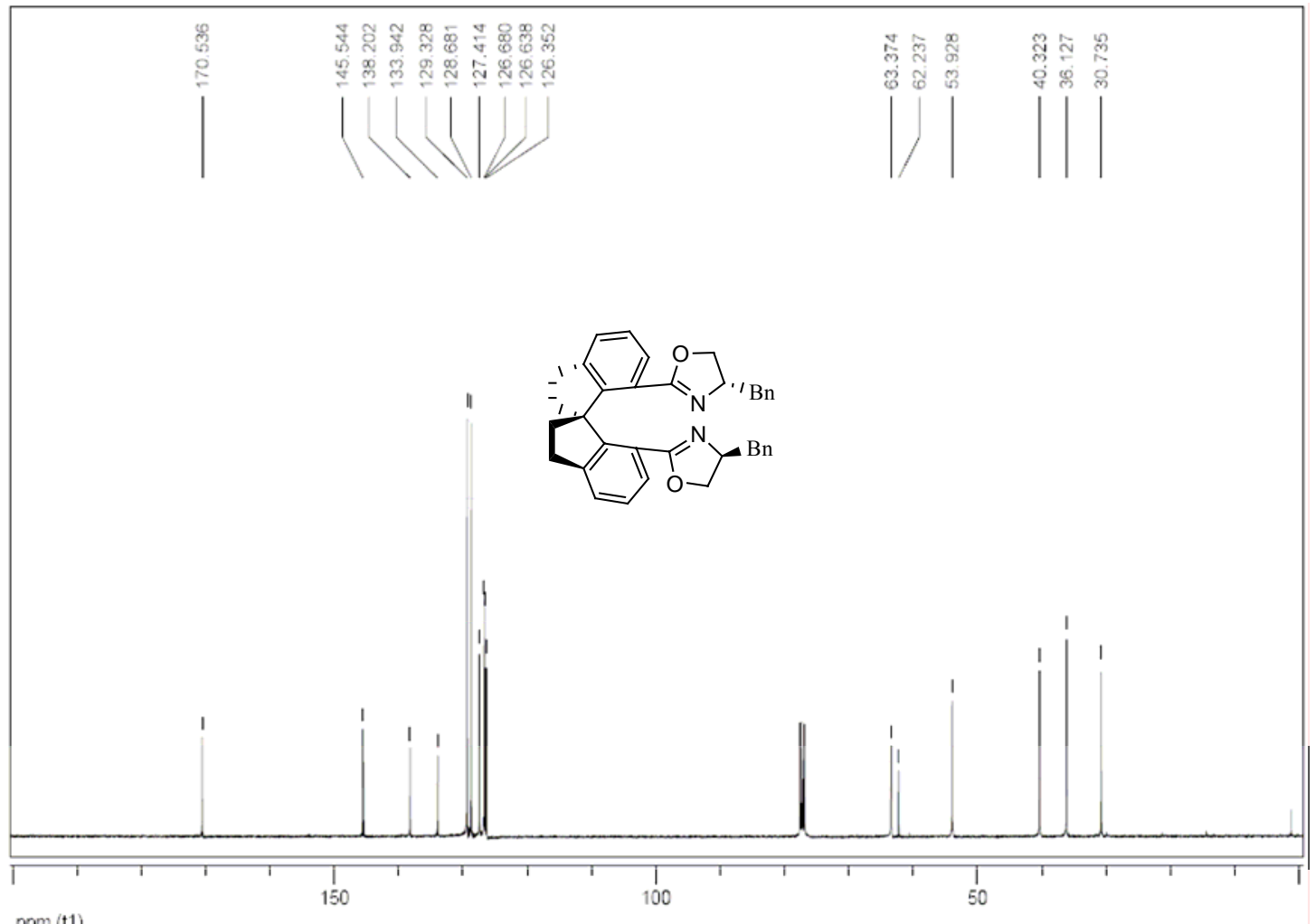


$\left(S_{a}, S, S\right)-7,7^{\prime}$-Bis(4-isopropyloxazolin-2-yl)-1,1'-spirobiindane
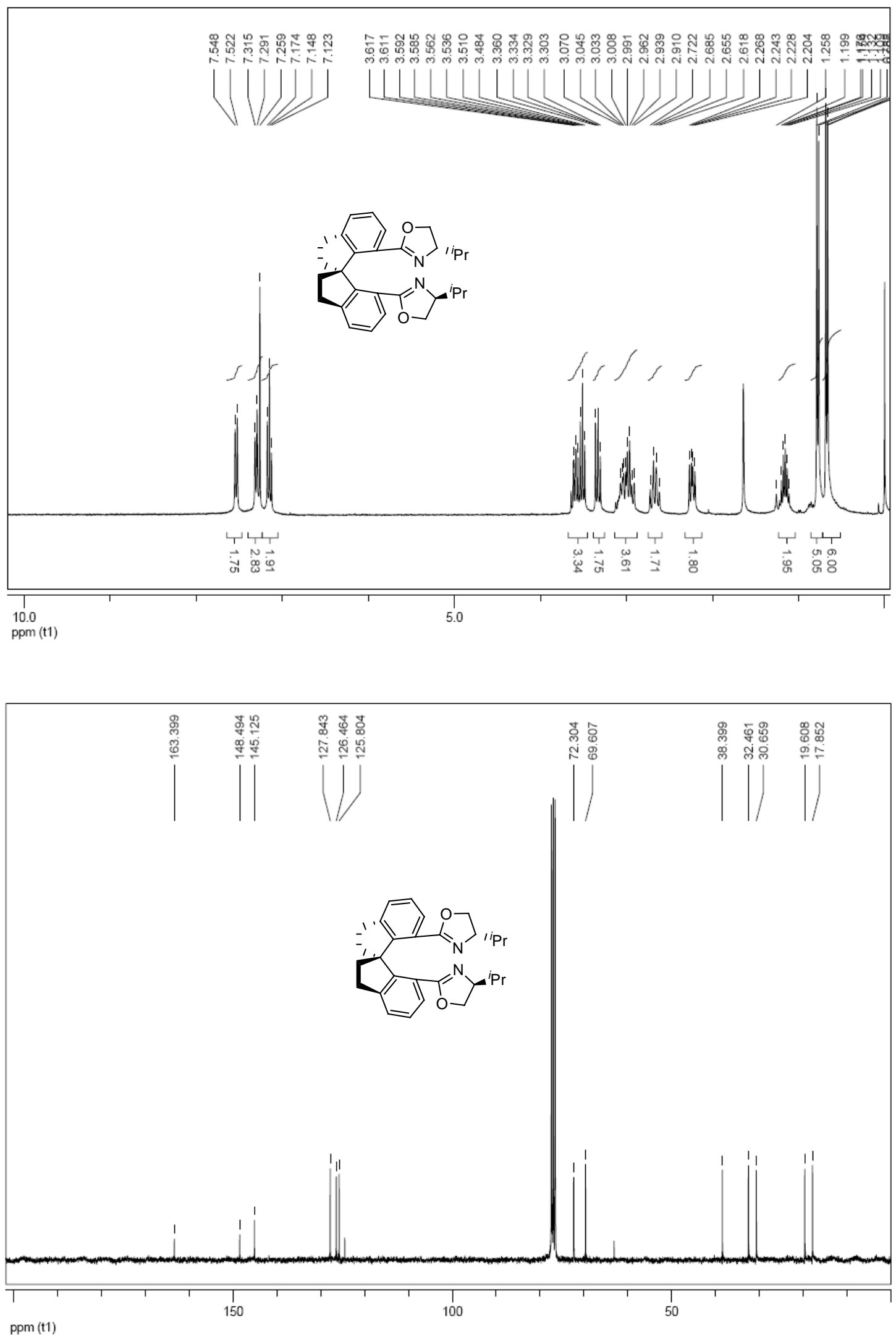
$\left(S_{a}, S, S\right)-7,7^{\prime}$-Bis (4-tert-butyloxazolin-2-yl)-1,1'-spirobiindane
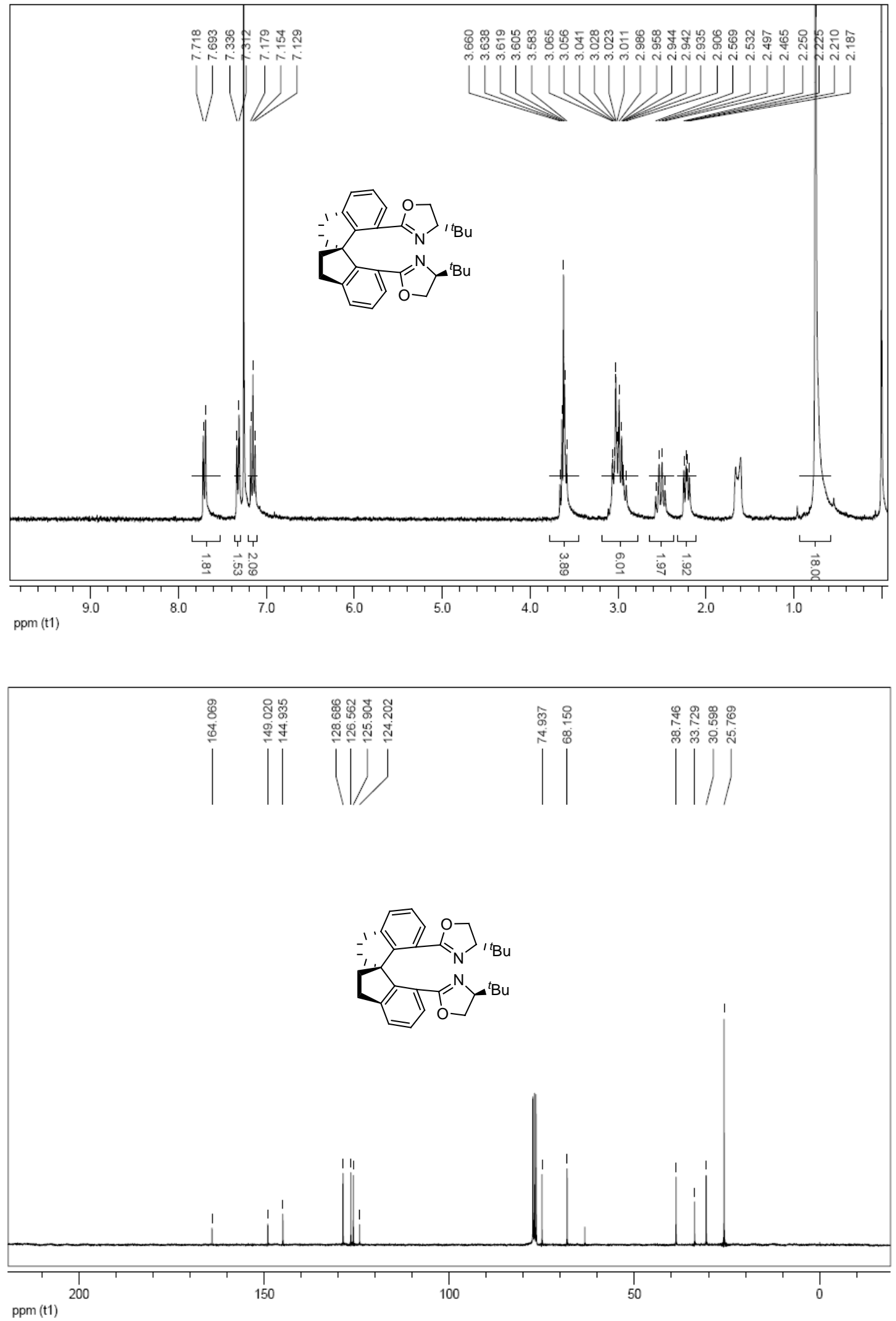
5. NMR Spectra for New N-H Insertion Products

(+)-Ethyl 2-(p-chlorophenylamino)propionate (4d)

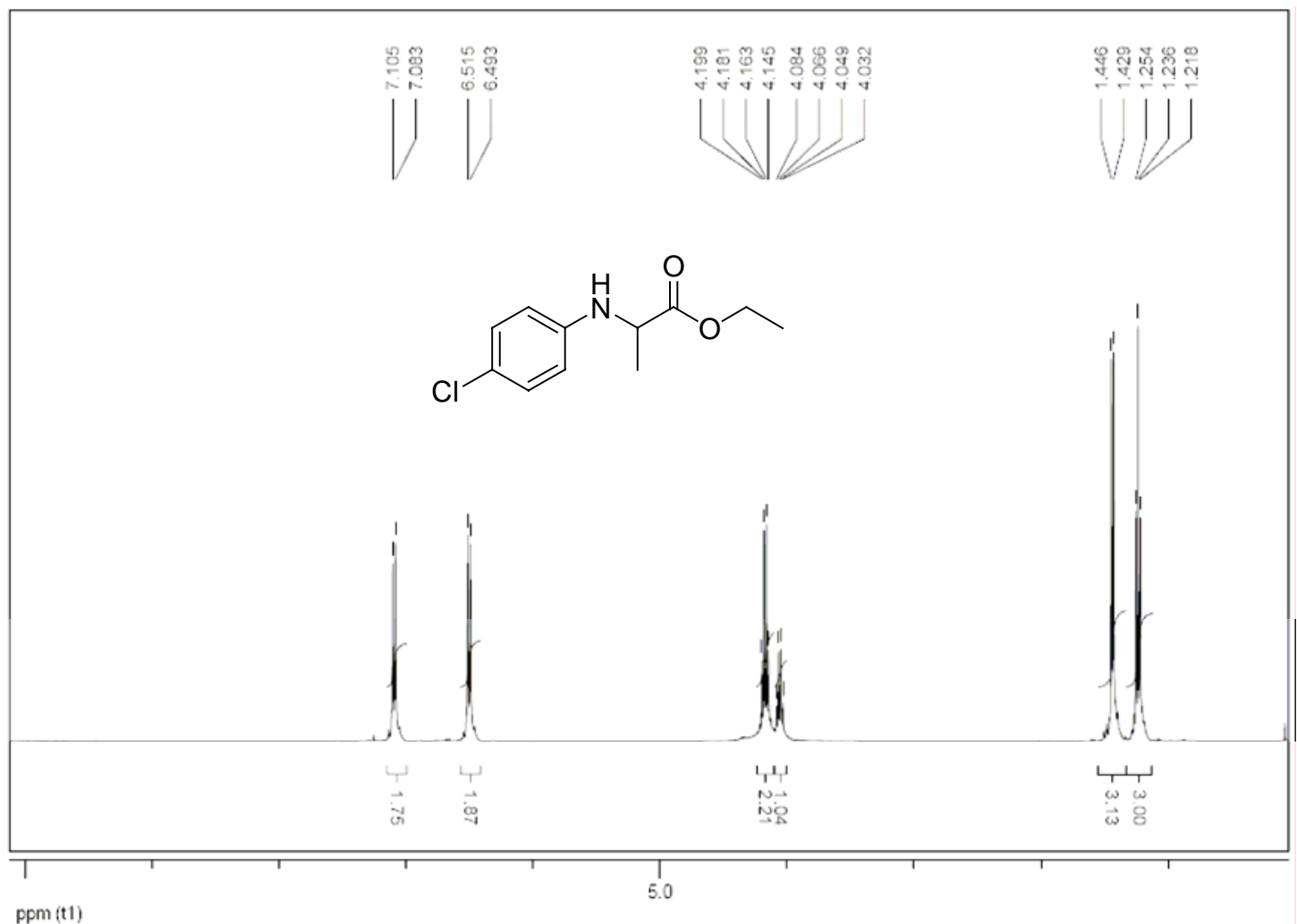

ppm (t1)

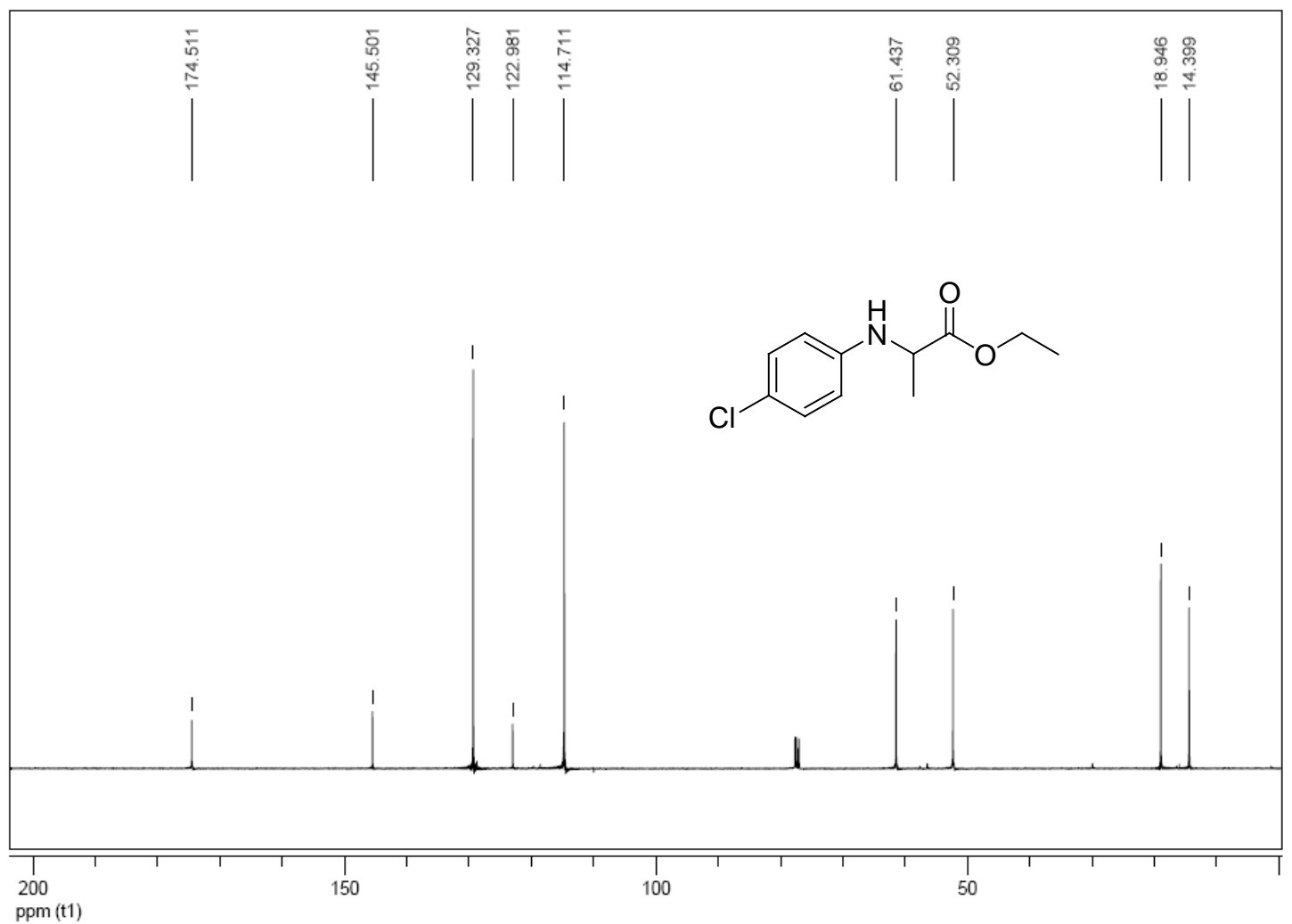


(+)-Ethyl 2-(p-bromophenylamino)propionate (4e)
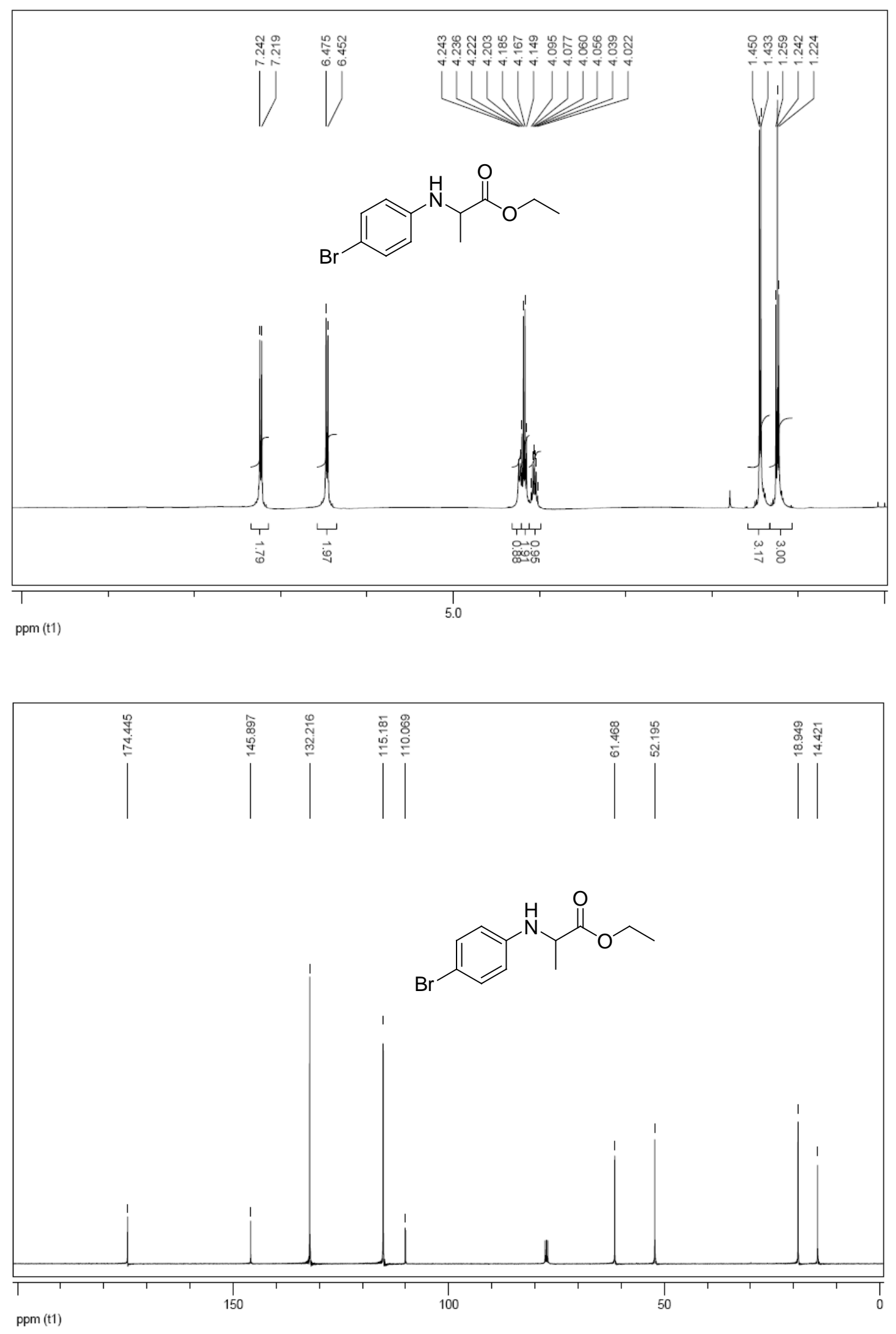

- S26 - 
(+)-Ethyl 2-(m-toluidino)propionate (4f)
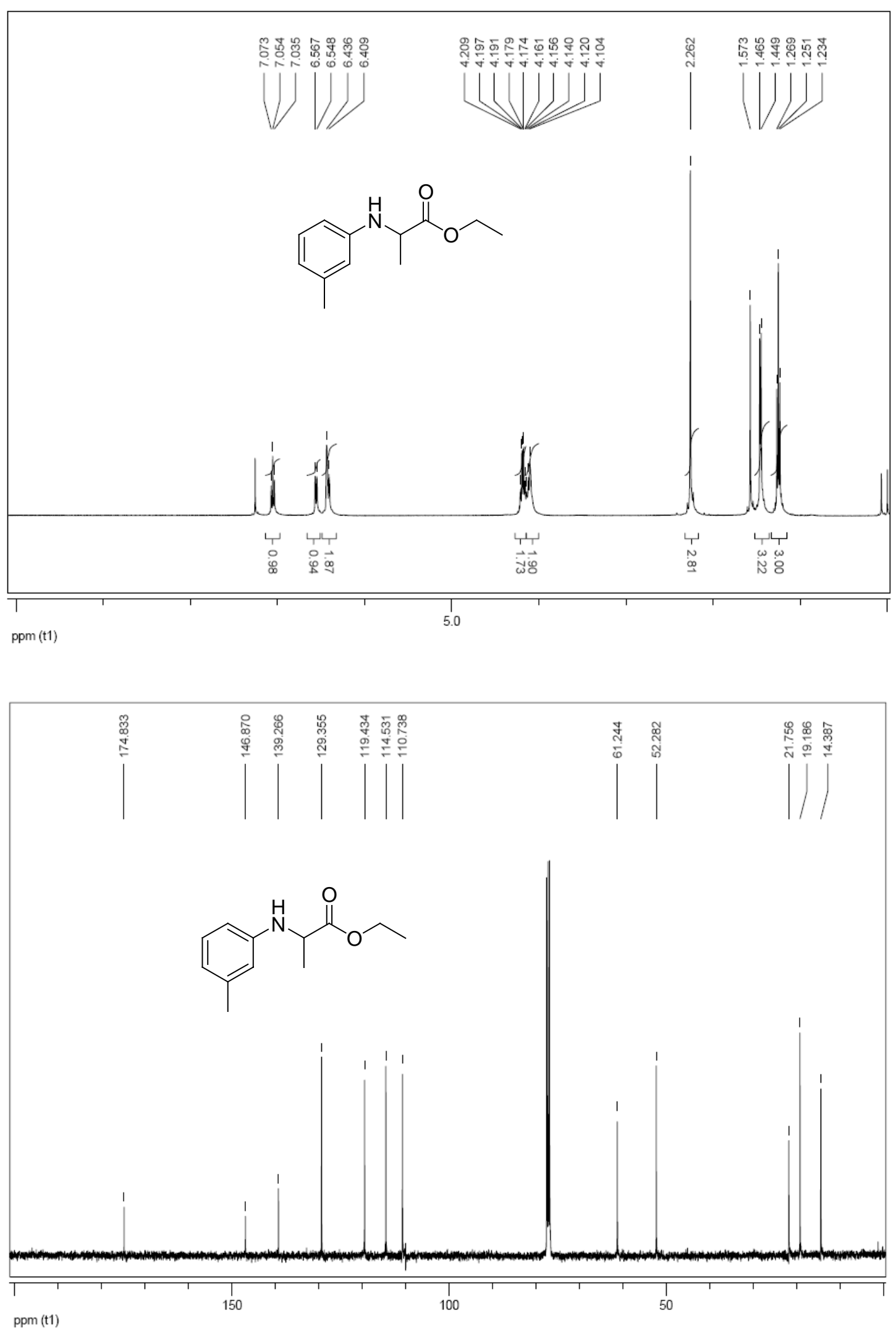
(+)-Ethyl 2-(m-bromophenylamino)propionate (4h)
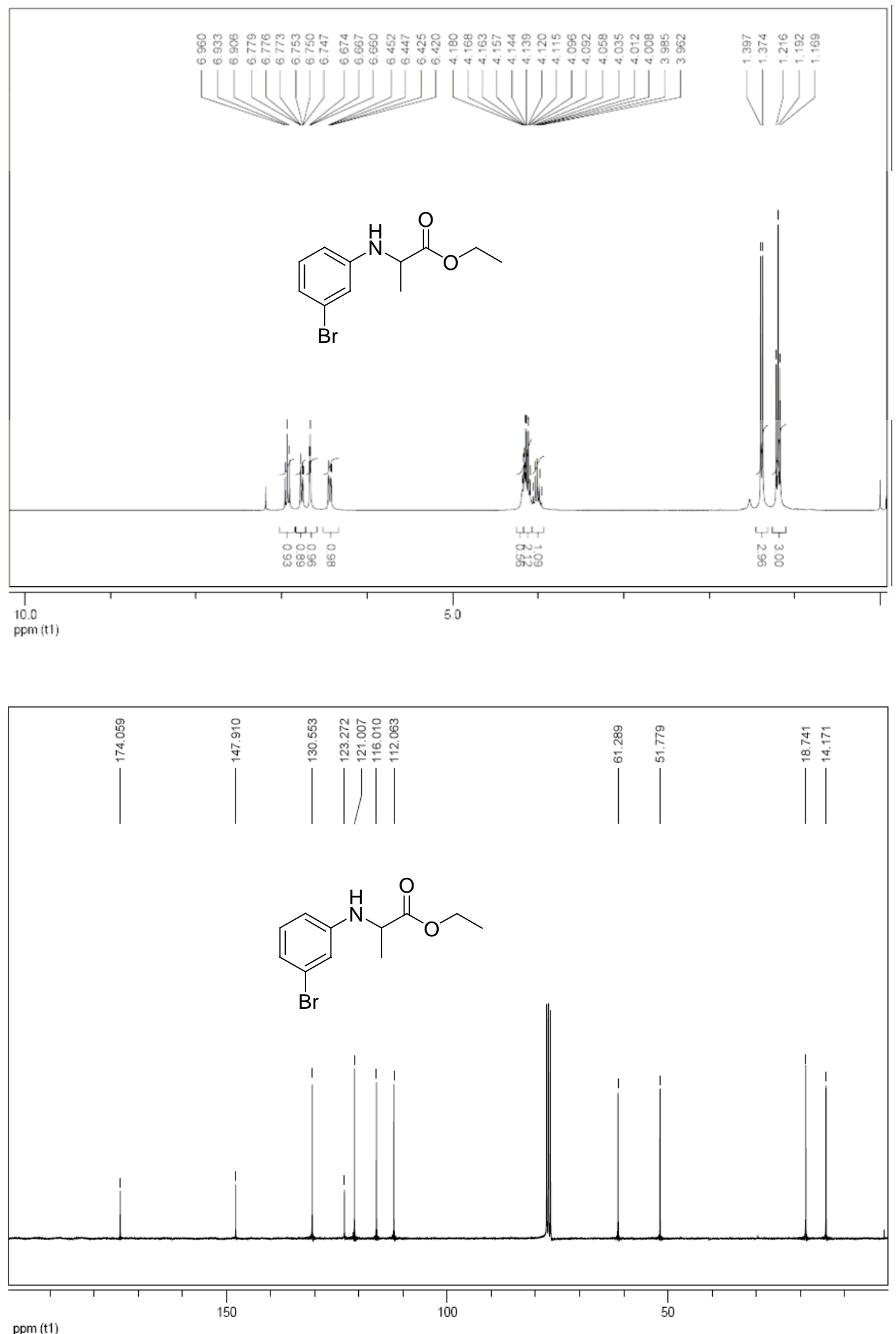
(+)-Ethyl 2-(o-toluidino)propionate (4i)
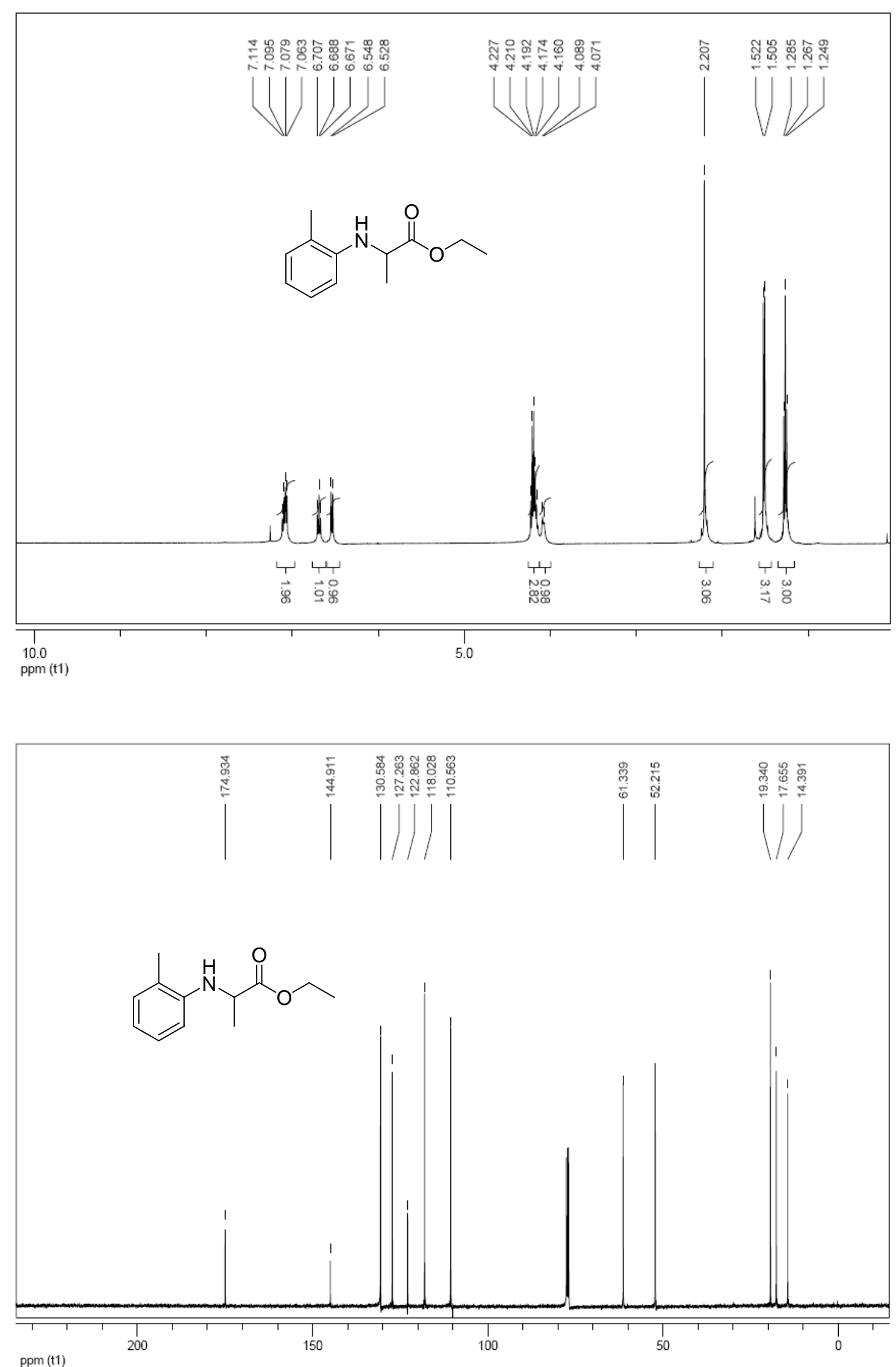
(+)-Ethyl 2-(o-chlorophenylamino)propionate (4k)
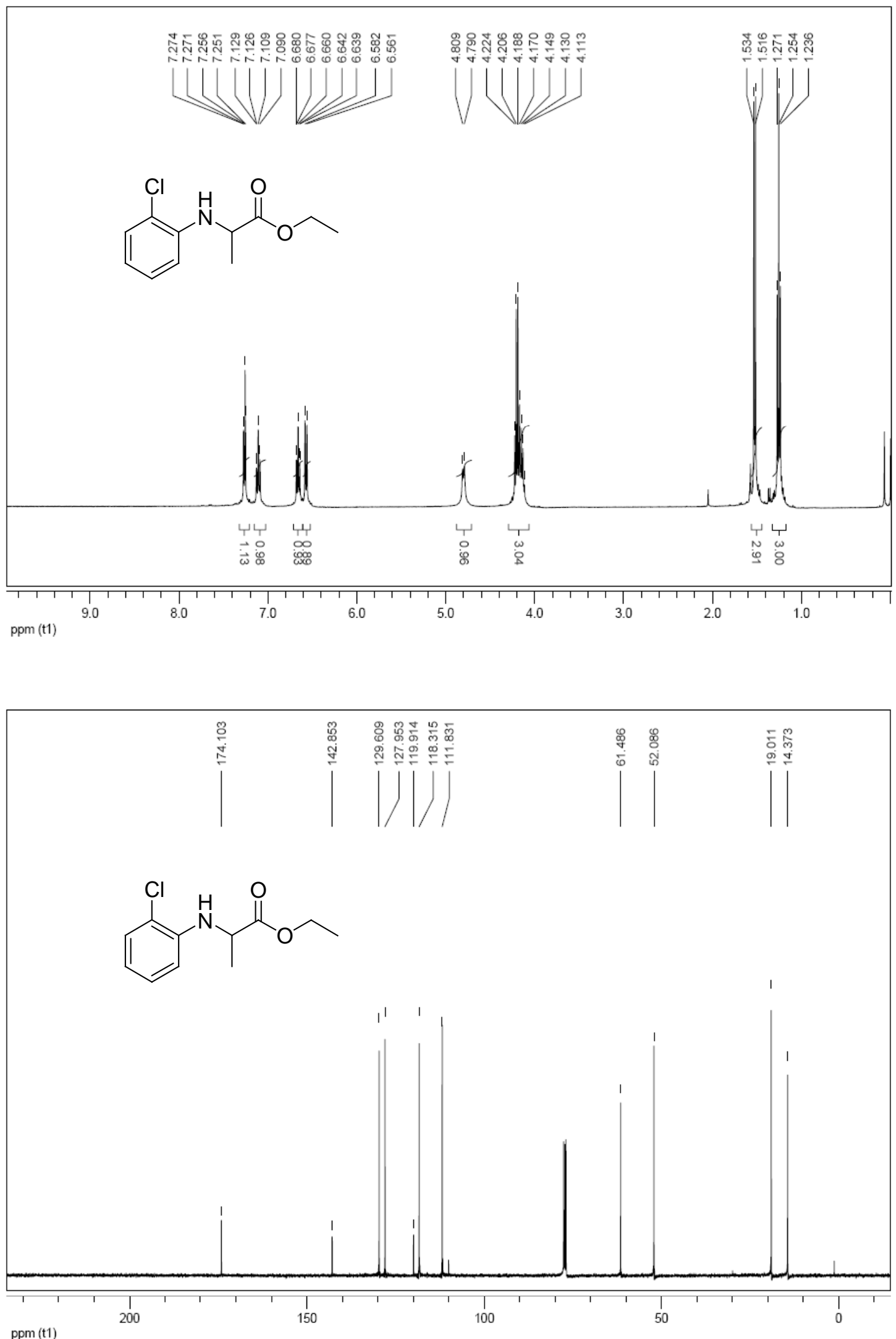
(+)-Ethyl 2-(naphthalen-2-ylamino)propionate (4m)
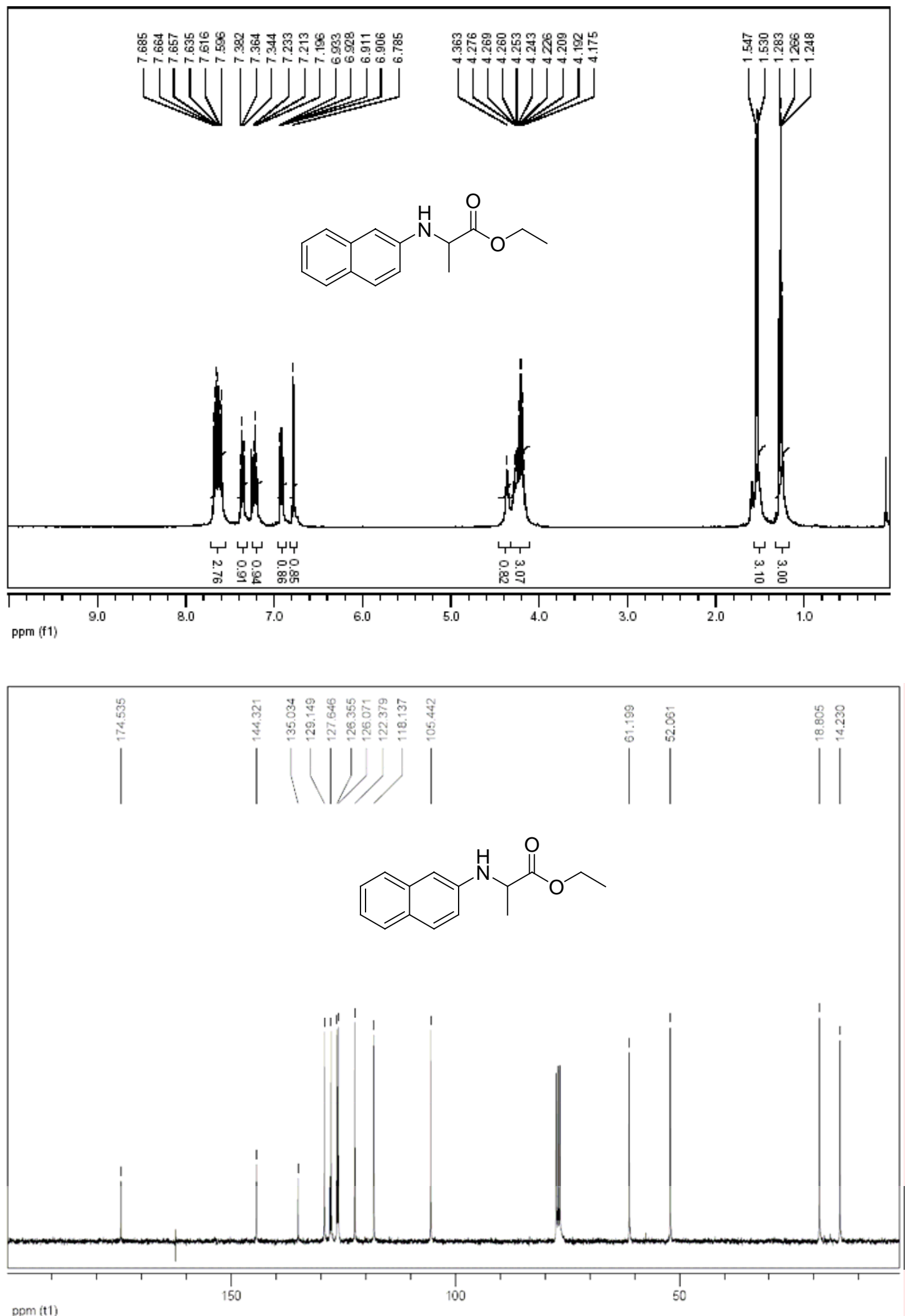
(+)-Ethyl 2-(phenylamino)butyrate (4p)

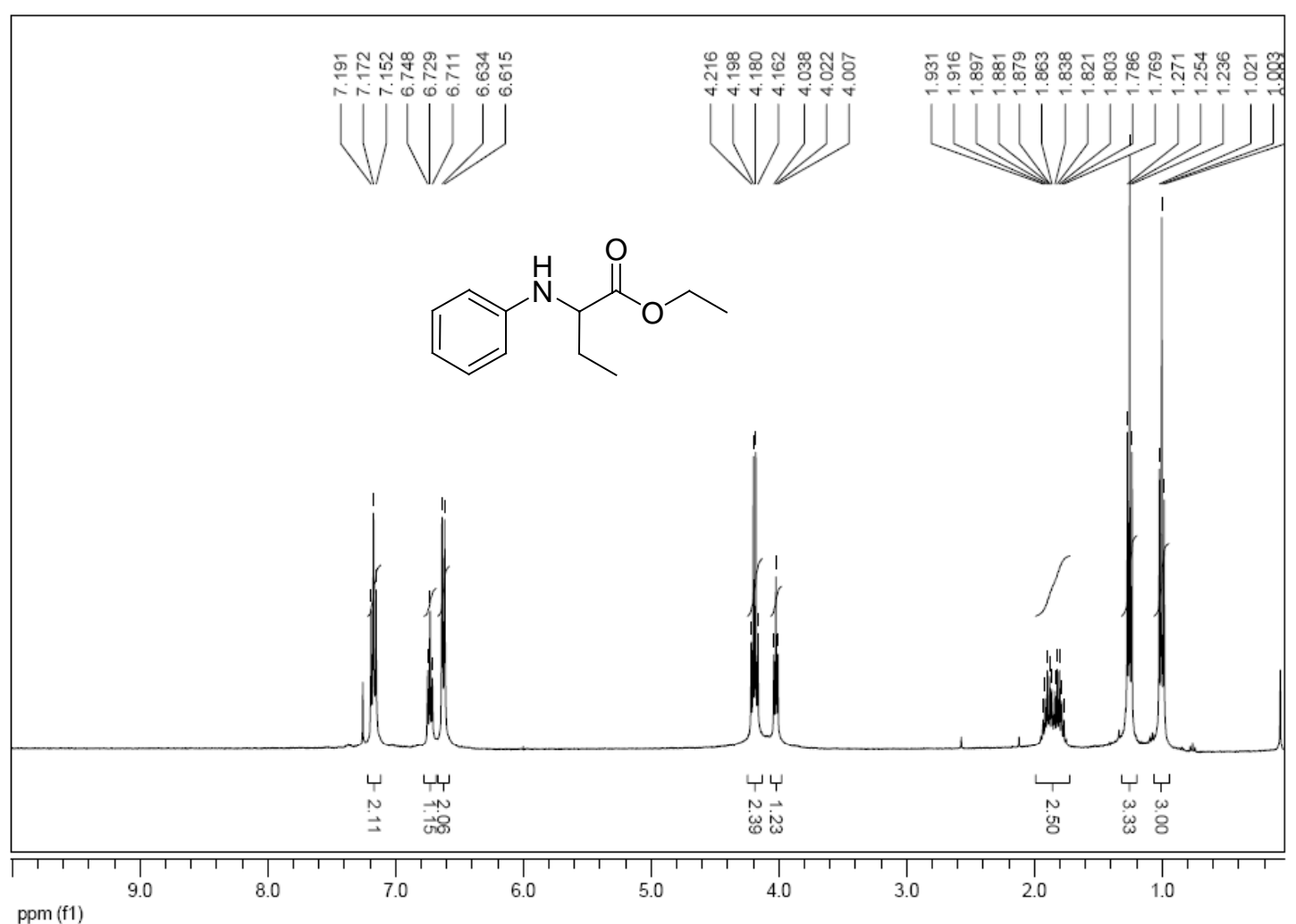

ppm (f1)

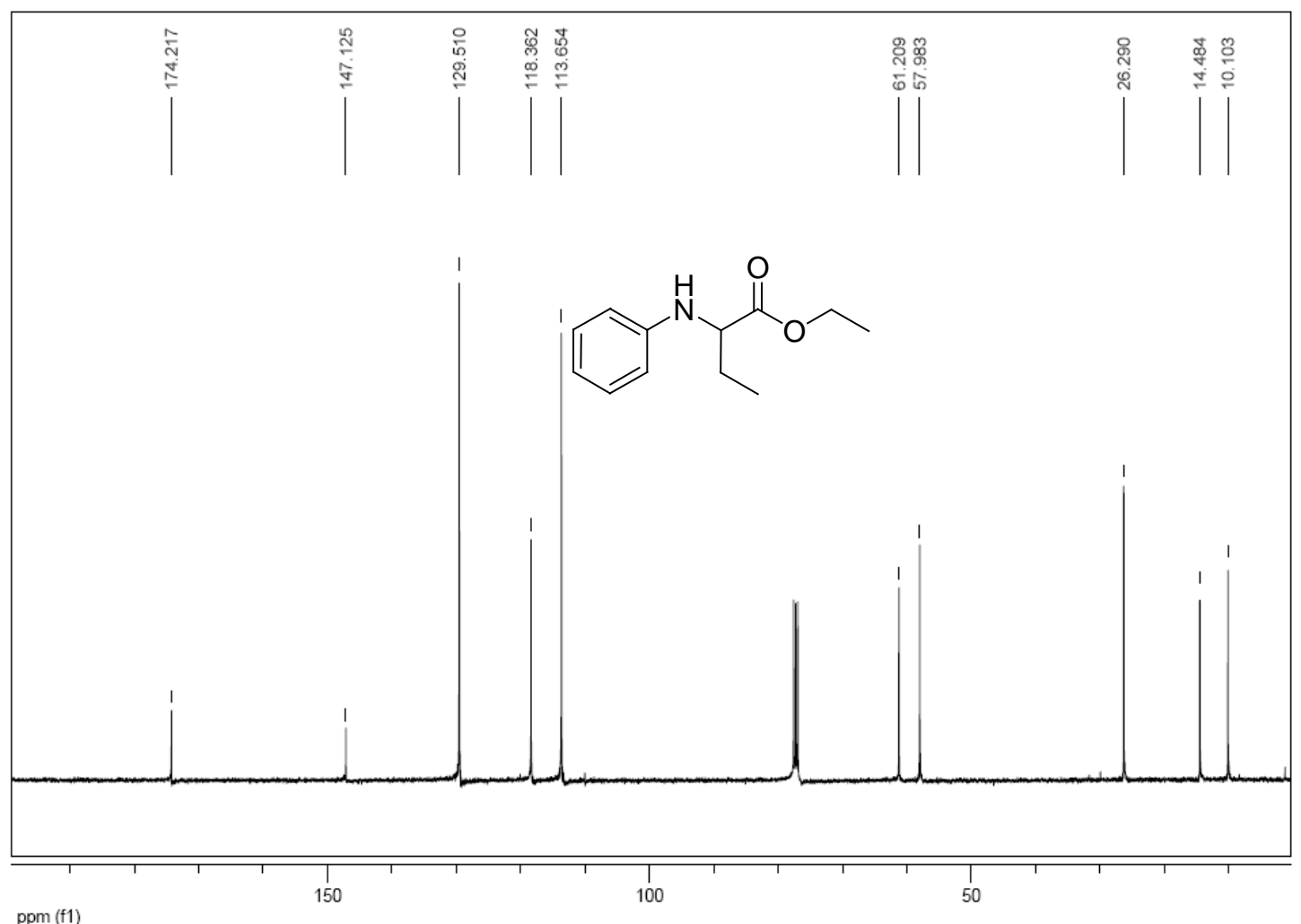




\section{HPLC Charts for N-H Insertion Products}

(+)-Ethyl 2-(phenylamino)propionate (4a)
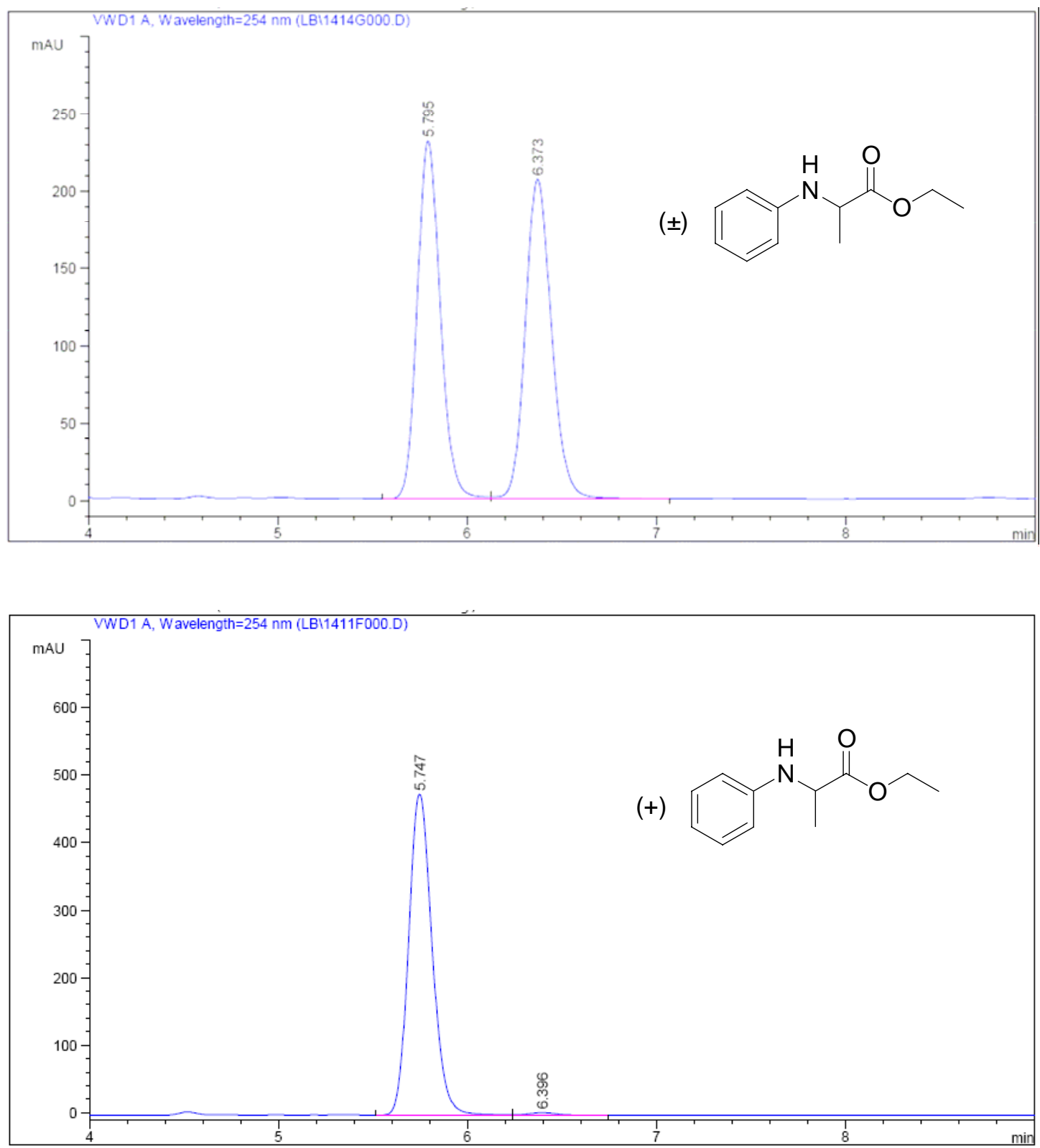

Signal 1: VWD1 A, Wavelength=254 nm

\begin{tabular}{|c|c|c|c|c|c|c|}
\hline eak & RetTime & Type & Width & Area & Height & Area \\
\hline$\#$ & [min] & & [min] & $\mathrm{mAU} \quad{ }^{\star} \mathrm{s}$ & {$[\mathrm{mAU} \quad]$} & 8 \\
\hline 1 & & KV & 360 & 14.32178 & 475 & \\
\hline 2 & .396 & VB & & 44.32206 & 4.11814 & 1.0658 \\
\hline
\end{tabular}




\section{(+)-Ethyl 2-(p-toluidino)propionate (4b)}
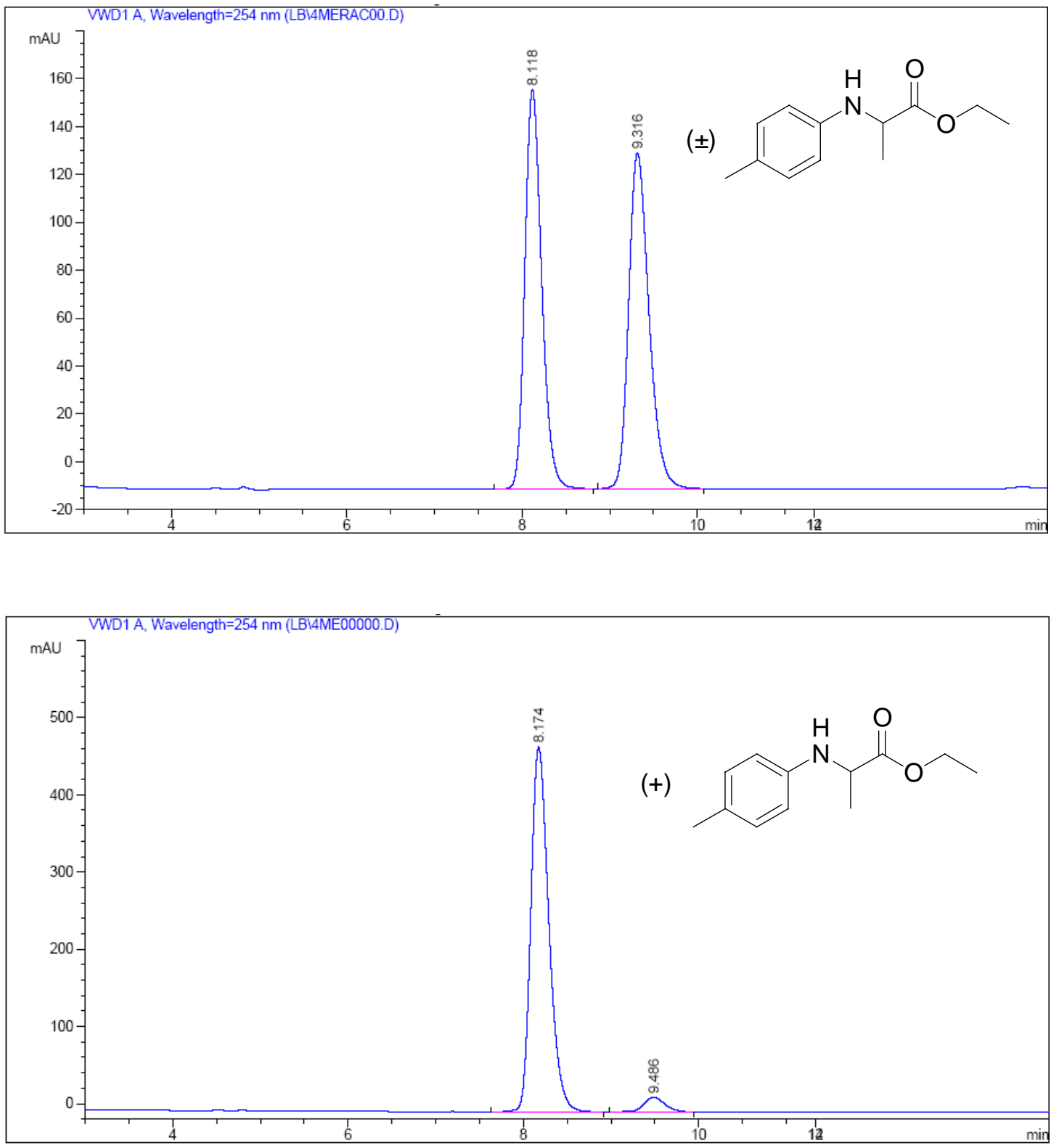

Signal 1: VWD1 A, Wavelength=254 nm

\begin{tabular}{|c|c|c|c|c|c|c|c|}
\hline eak & RetTime & Type & & & ea & Height & Area \\
\hline$\#$ & [min] & & [min] & MAU & ${ }^{\star} \mathrm{s}$ & {$[\mathrm{mAU} \quad]$} & 8 \\
\hline & & $\begin{array}{l}B B \\
B B\end{array}$ & & $\begin{array}{r}671 \\
30\end{array}$ & 06802 & $\begin{array}{r}472.6950 \\
18.6029\end{array}$ & $\begin{array}{r}95.6029 \\
4.3971\end{array}$ \\
\hline
\end{tabular}


(+)-Ethyl 2-(4-methoxyphenylamino)propionate (4c)
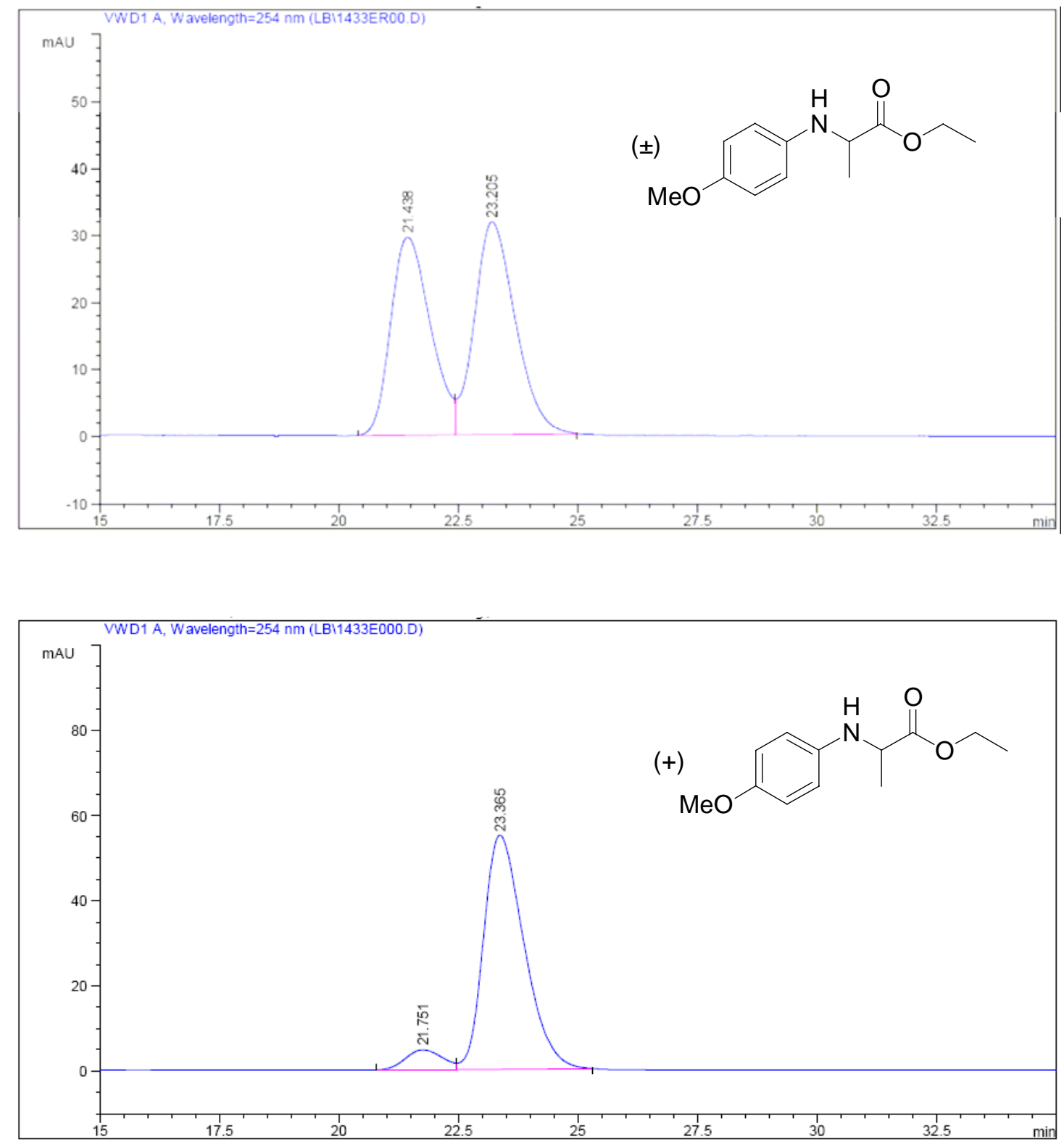

Signal 1: VWD1 A, Wavelength=254 nm

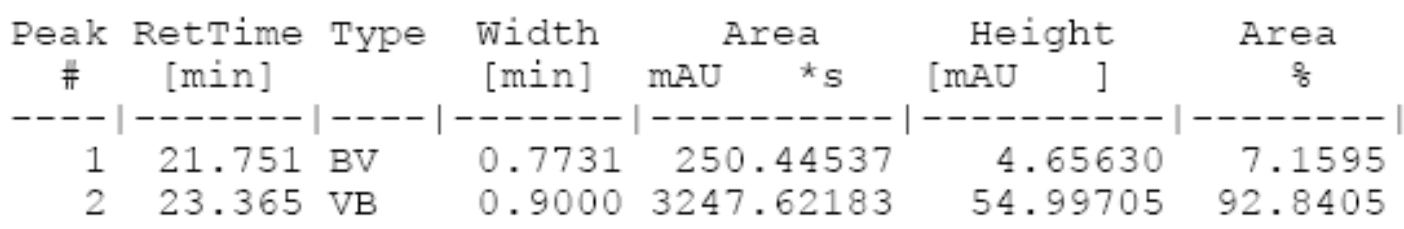


(+)-Ethyl 2-(p-chlorophenylamino)propionate (4d)
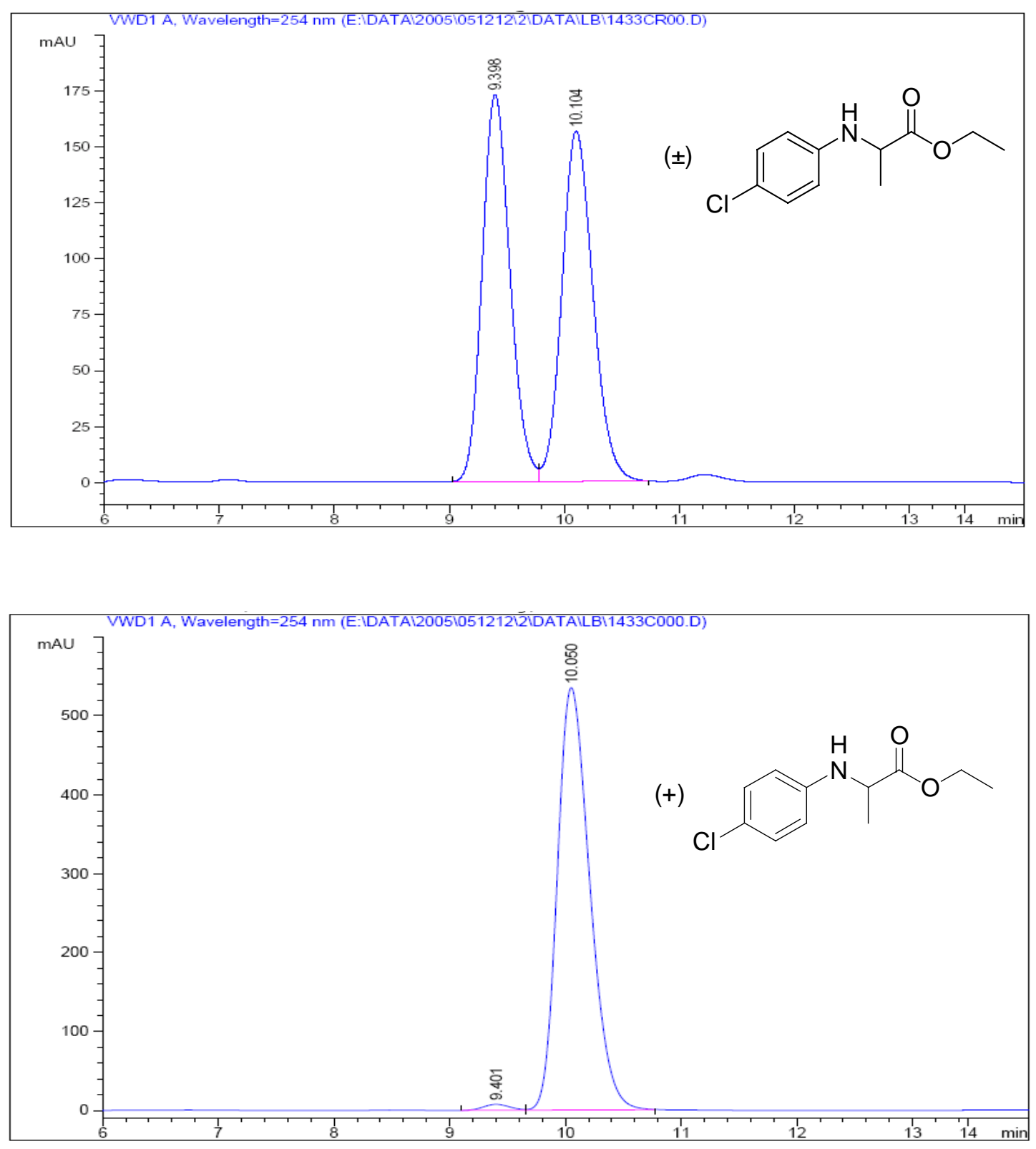

Signal 1: VWD1 A, Wavelength=254 nm

\begin{tabular}{|c|c|c|c|c|c|c|}
\hline Peak & Retrime & Type & width & Area & Height & Area \\
\hline \# & [min] & & [min] & $\mathrm{mAU} \quad{ }^{\prime} \mathrm{s}$ & {$[\mathrm{mAU} \quad]$} & $\frac{8}{8}$ \\
\hline $\begin{array}{l}1 \\
2\end{array}$ & $\begin{array}{r}9.401 \\
10.050\end{array}$ & $\begin{array}{l}\text { PV } \\
\text { VB }\end{array}$ & $\begin{array}{l}0.2502 \\
0.3017\end{array}$ & $\begin{array}{c}120.26642 \\
1.04438 \mathrm{e} 4\end{array}$ & $\begin{array}{r}7.53814 \\
535.74323\end{array}$ & $\begin{array}{r}1.1384 \\
98.8616\end{array}$ \\
\hline
\end{tabular}




\section{(+)-Ethyl 2-(p-bromophenylamino)propionate (4e)}
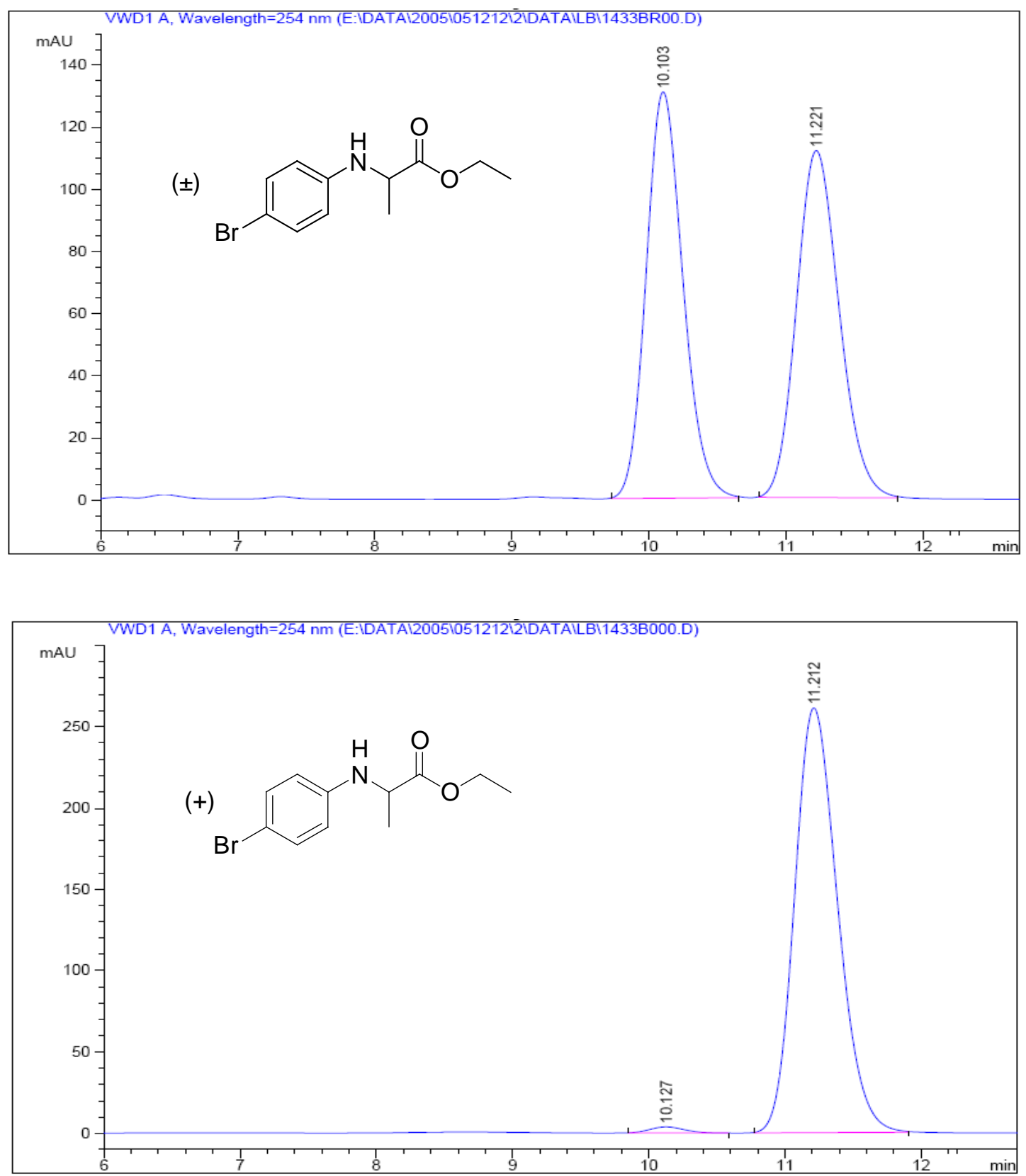

Signal 1: VWD1 A, Wavelength=254 nm

\begin{tabular}{|c|c|c|c|c|c|c|}
\hline Peak & RetTime & Type & width & Area & Height & Area \\
\hline \# & [min] & & [min] & $\mathrm{mAU} \quad * \mathrm{~s}$ & {$[\mathrm{mAU}]$} & $\frac{9}{6}$ \\
\hline & & & & $\mid---------$ & ---------- & \\
\hline 1 & 10.127 & $B P$ & & $\begin{array}{r}69.01994 \\
\end{array}$ & 613 & 864 \\
\hline 2 & & $\mathrm{BB}$ & 409 & 5748.64648 & 261.48798 & 8136 \\
\hline
\end{tabular}


(+)-Ethyl 2-(m-toluidino)propionate (4f)
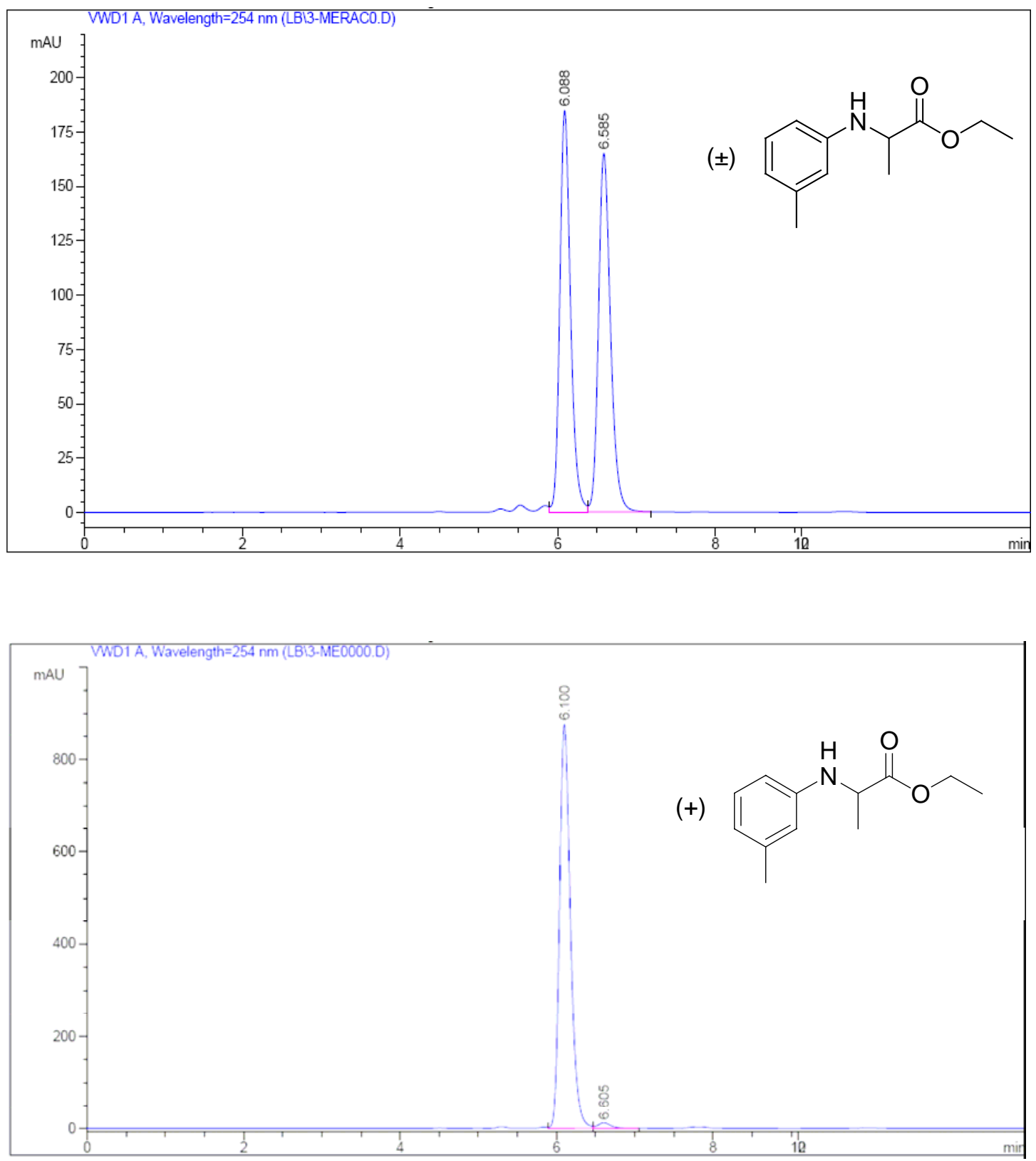

Signal 1: VWD1 A, Wavelength=254 nm

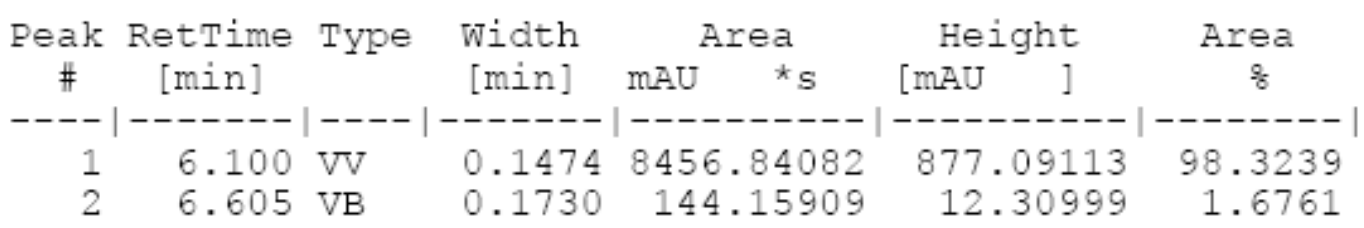


(+)-Ethyl 2-(m-chlorophenylamino)propionate (4g)
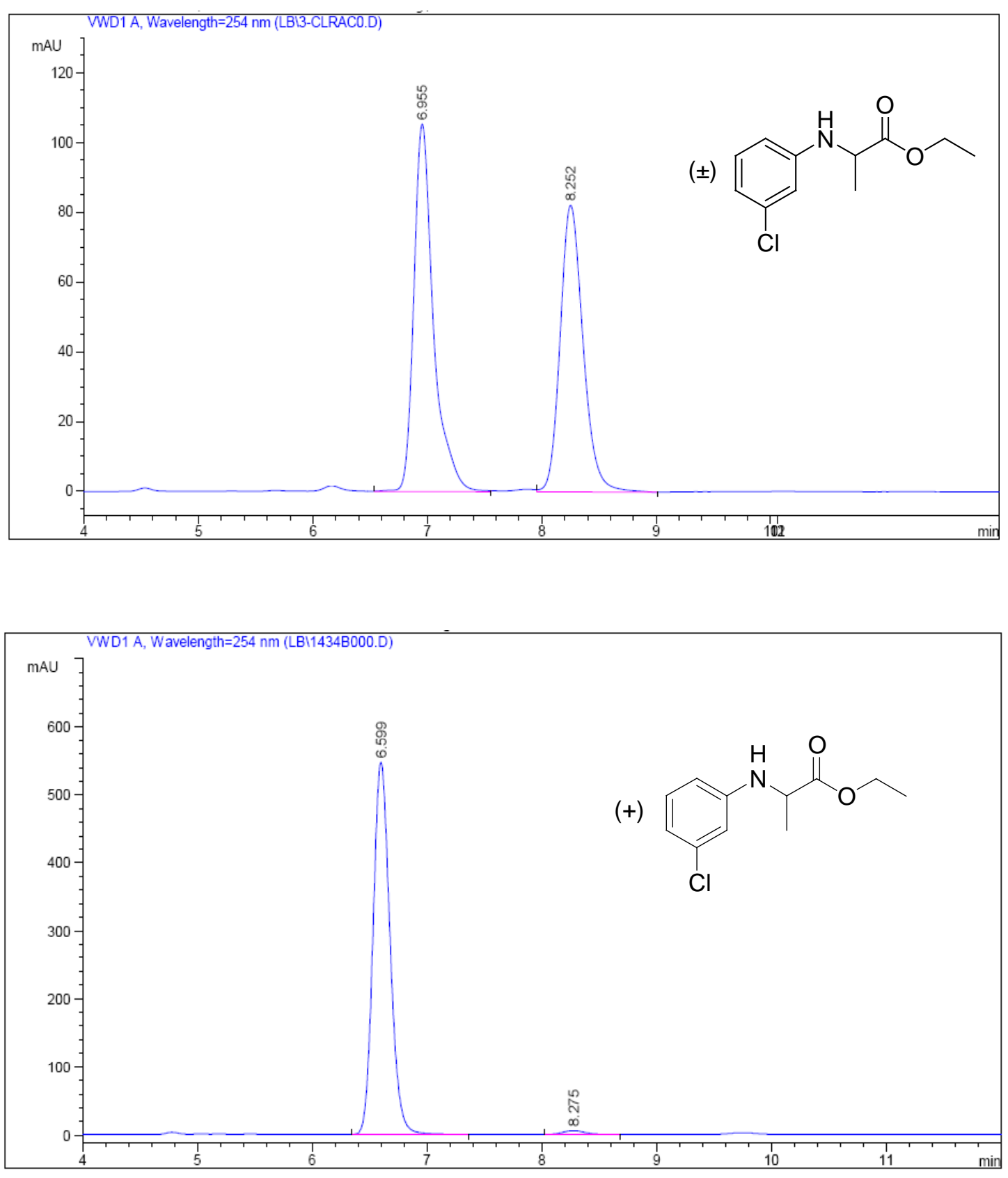

Signal 1: VWD1 A, Wavelength=254 nm

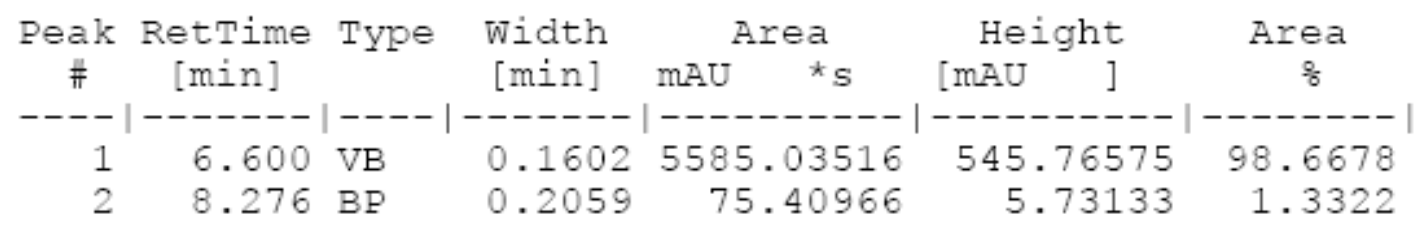


(+)-Ethyl 2-(m-bromophenylamino)propionate (4h)
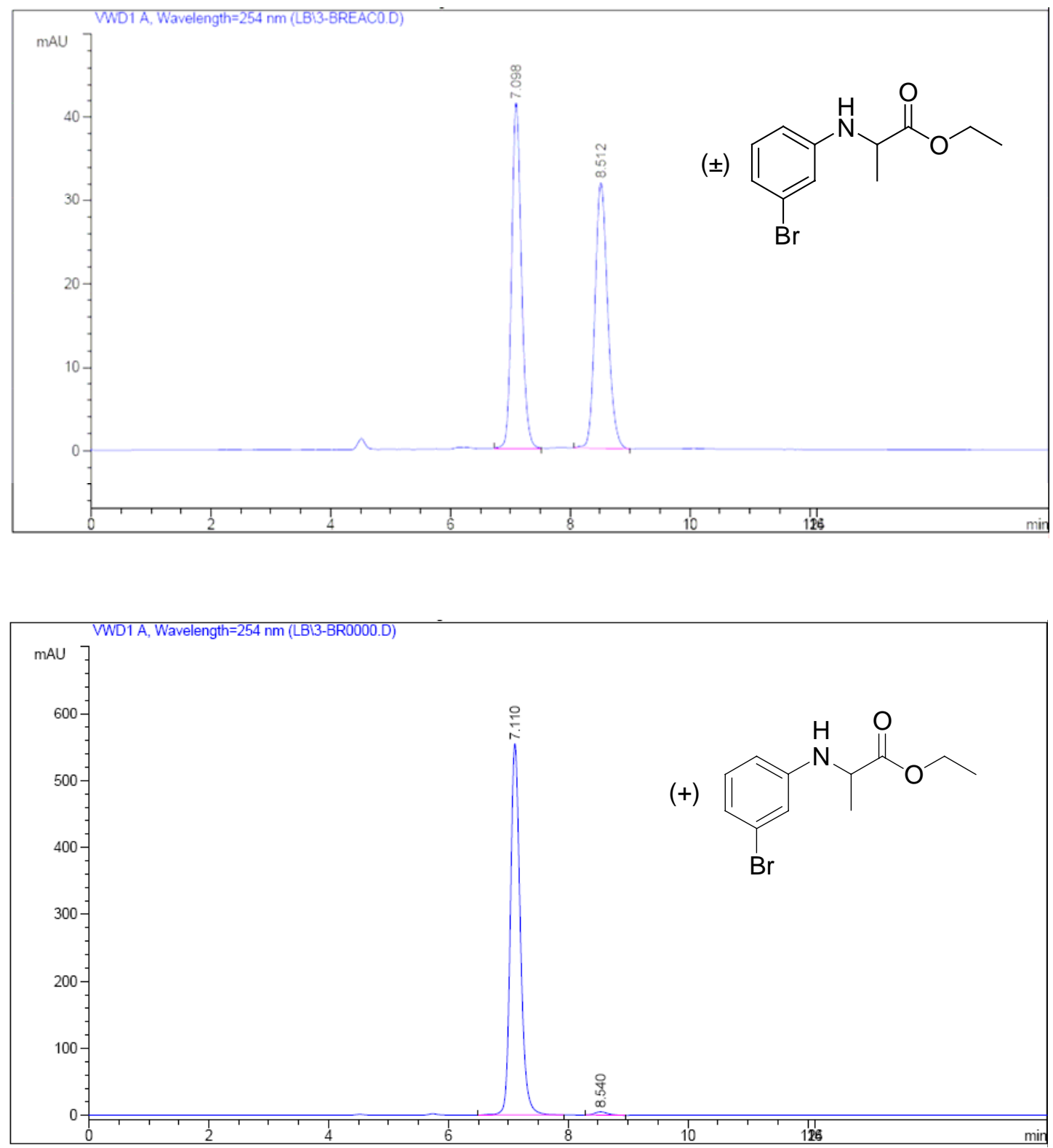

Signal 1: VWD1 A, Wavelength=254 nm

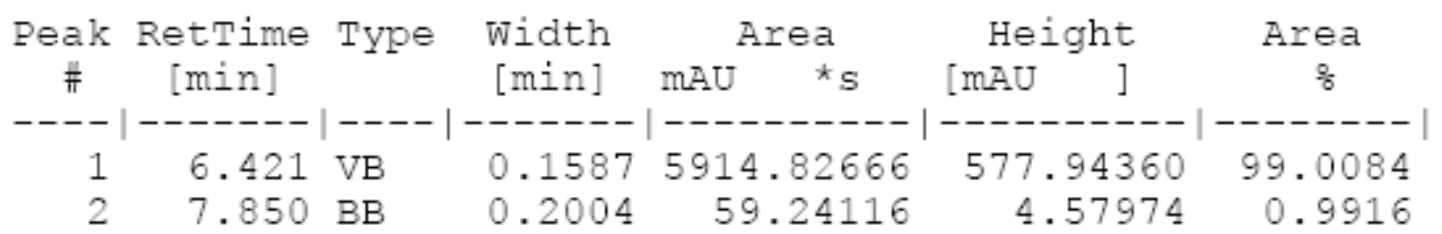


(+)-Ethyl 2-(o-toluidino)propionate (4i)
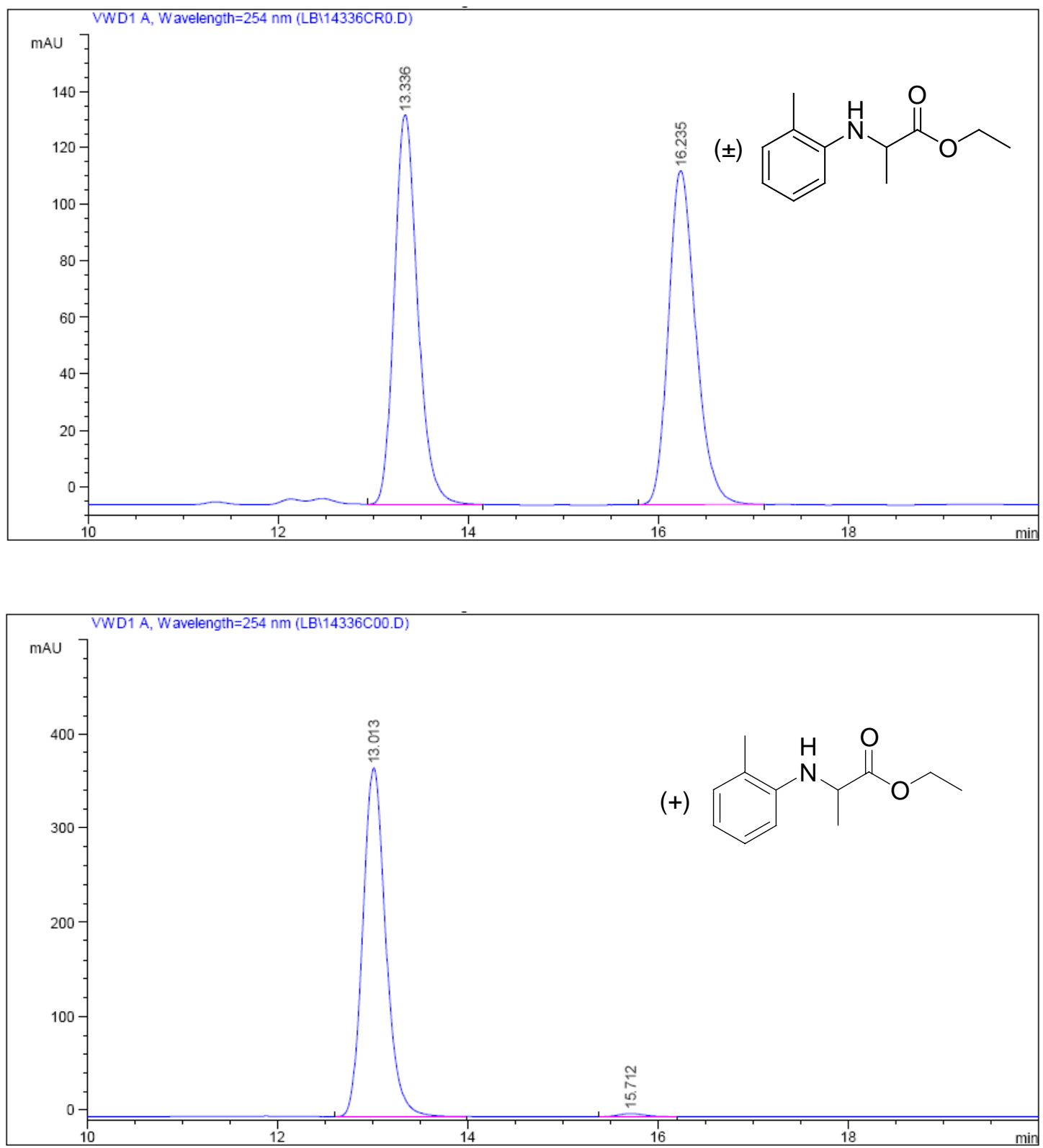

Signal 1: VWD1 A, Wavelength $=254 \mathrm{~nm}$

\begin{tabular}{|c|c|c|c|c|c|c|}
\hline Peak & RetTime & Type & Width & Area & Height & Area \\
\hline \# & [min] & & [min] & mAU $\quad * s$ & {$[\mathrm{mAU}]$} & 8 \\
\hline 1 & $-----\overline{13}$ & $P B$ & 0.2641 & 6214.55713 & 370.52734 & $\begin{array}{l}----1 \\
9876\end{array}$ \\
\hline 2 & 15.712 & $\mathrm{BB}$ & 0.2846 & 63.56122 & 3.36291 & 1.0124 \\
\hline
\end{tabular}


(+)-Ethyl 2-(o-methoxyphenylamino)propionate (4j)
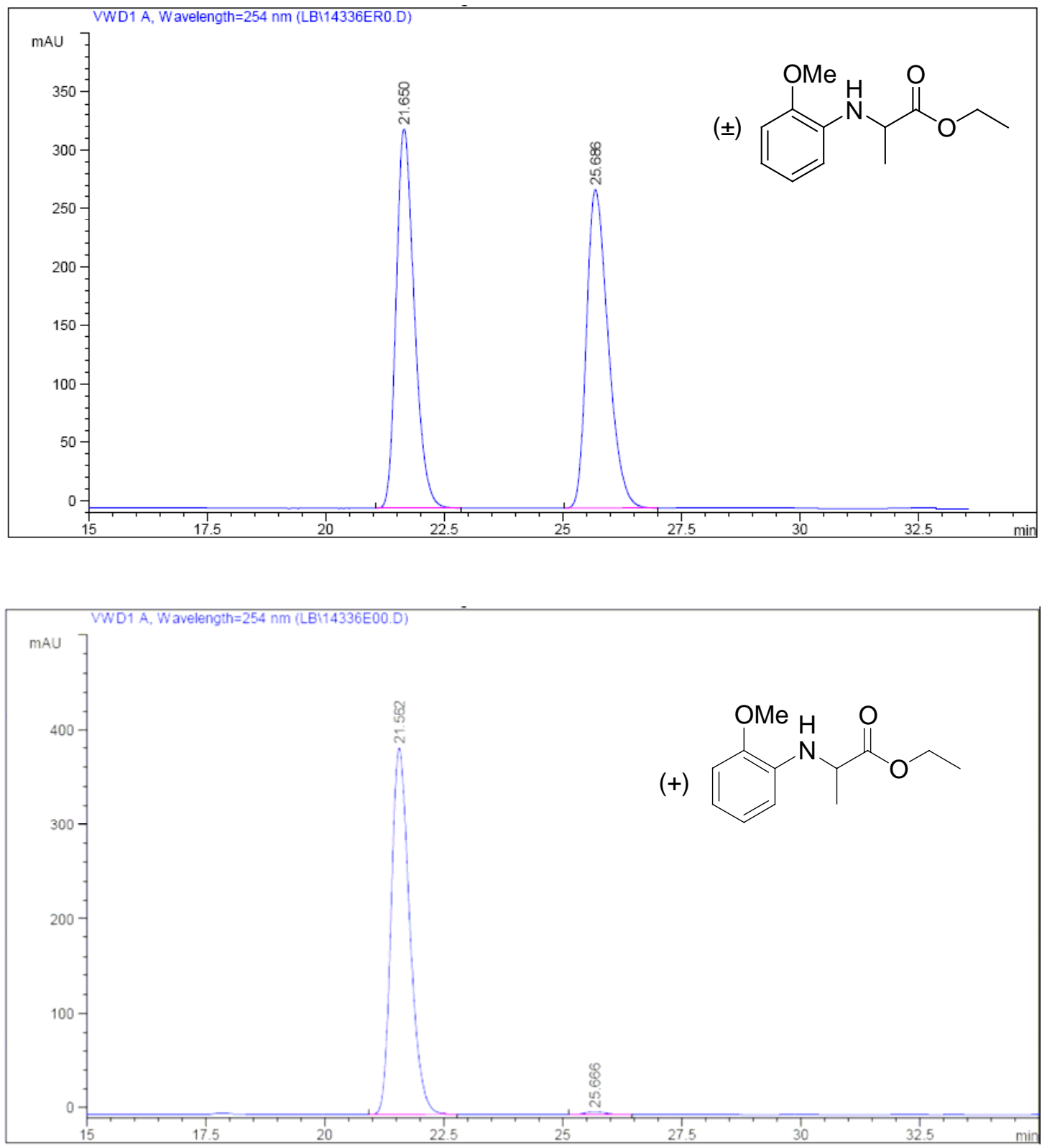

Signal 1: VWD1 A, Wavelength=254 nm

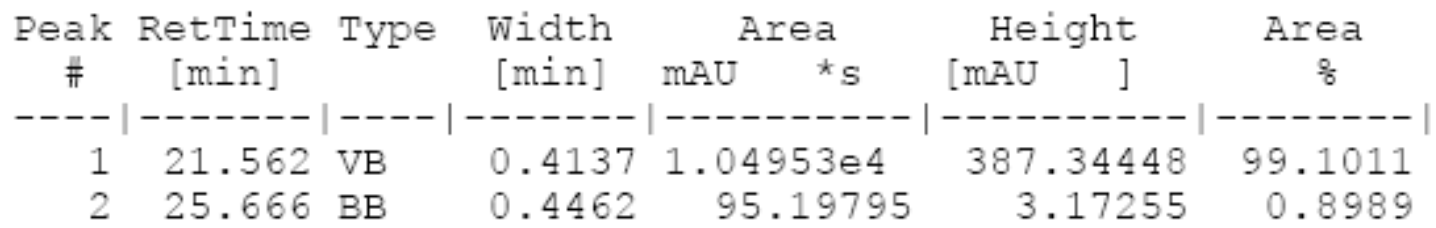


(+)-Ethyl 2-(o-fluorophenylamino)propionate (4k)
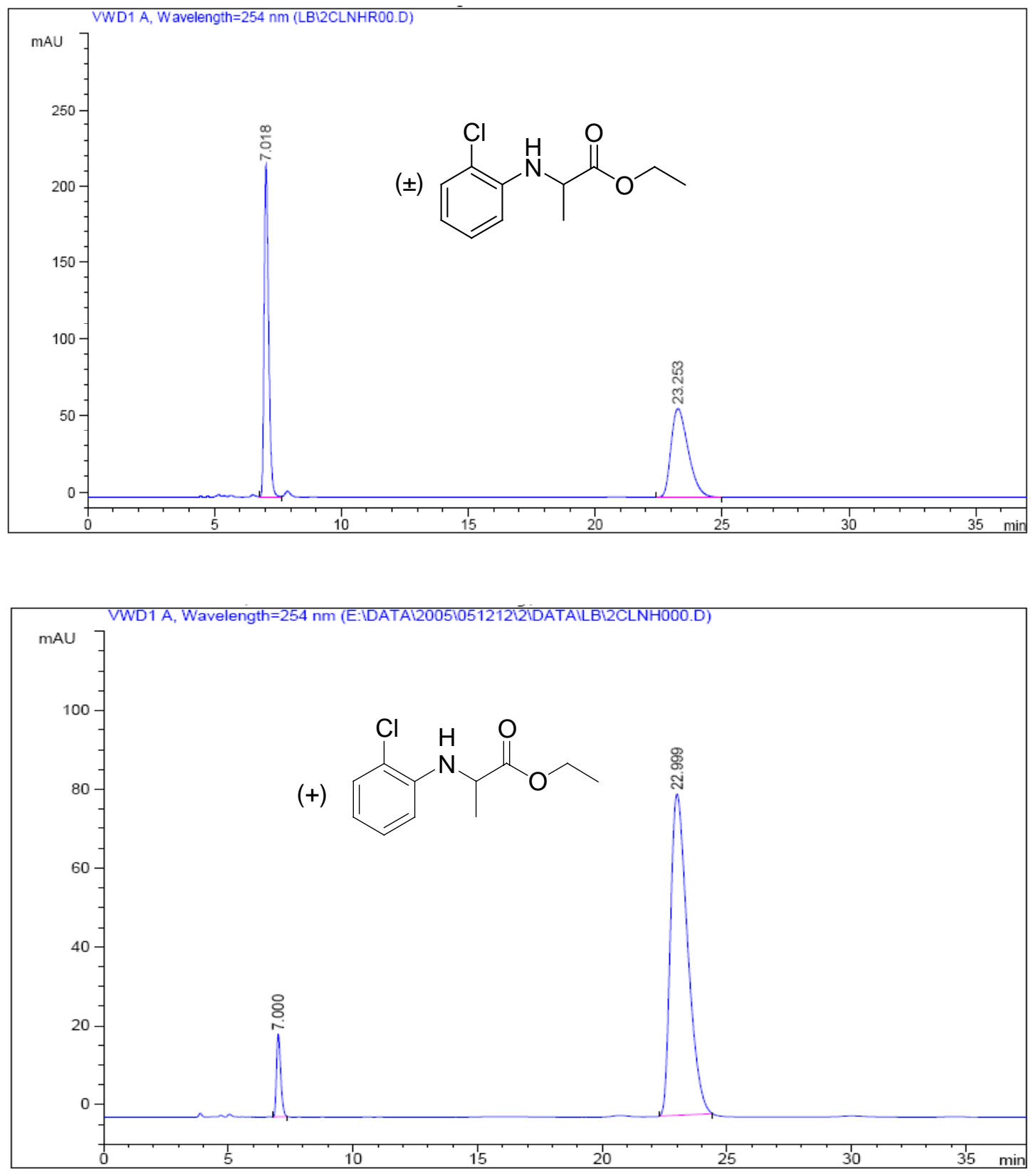

Signal 1: VWD1 A, Wavelength=254 nm

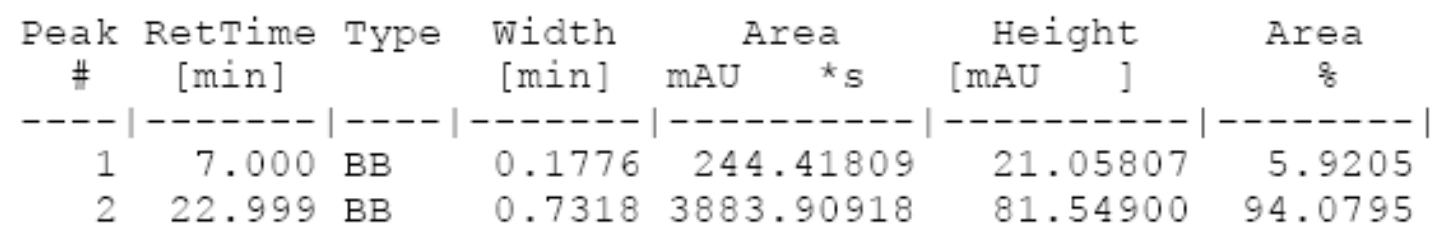




\section{(-)-Ethyl 2-(naphthalen-1-ylamino)propionate (4I)}
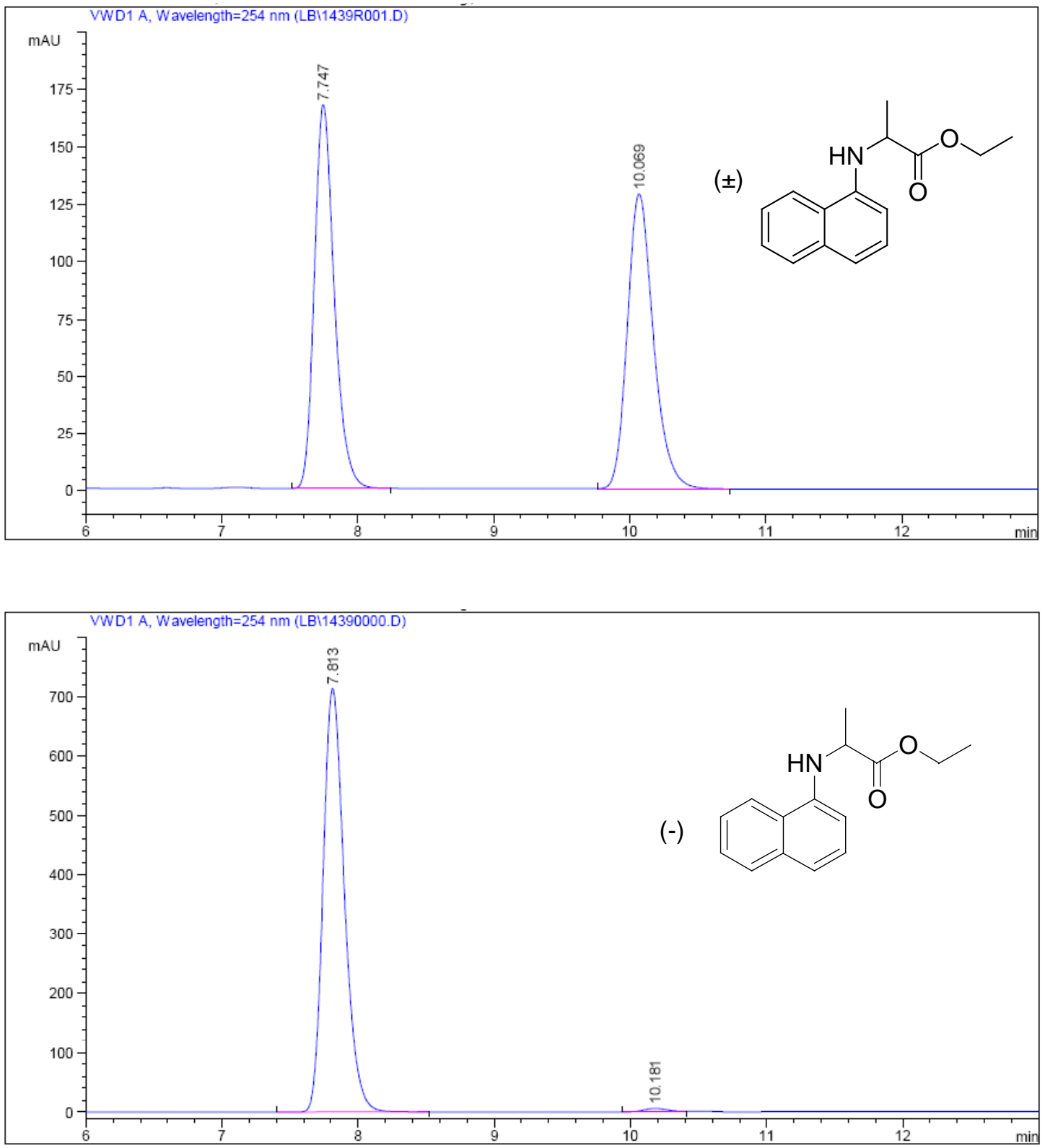

Signal 1: VWD1 A, Wavelength=254 nm

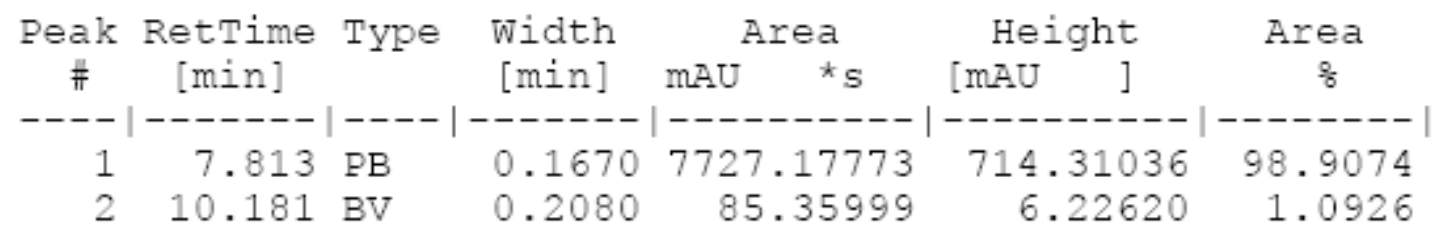


(+)-Ethyl 2-(naphthalen-2-ylamino)propionate (4m)
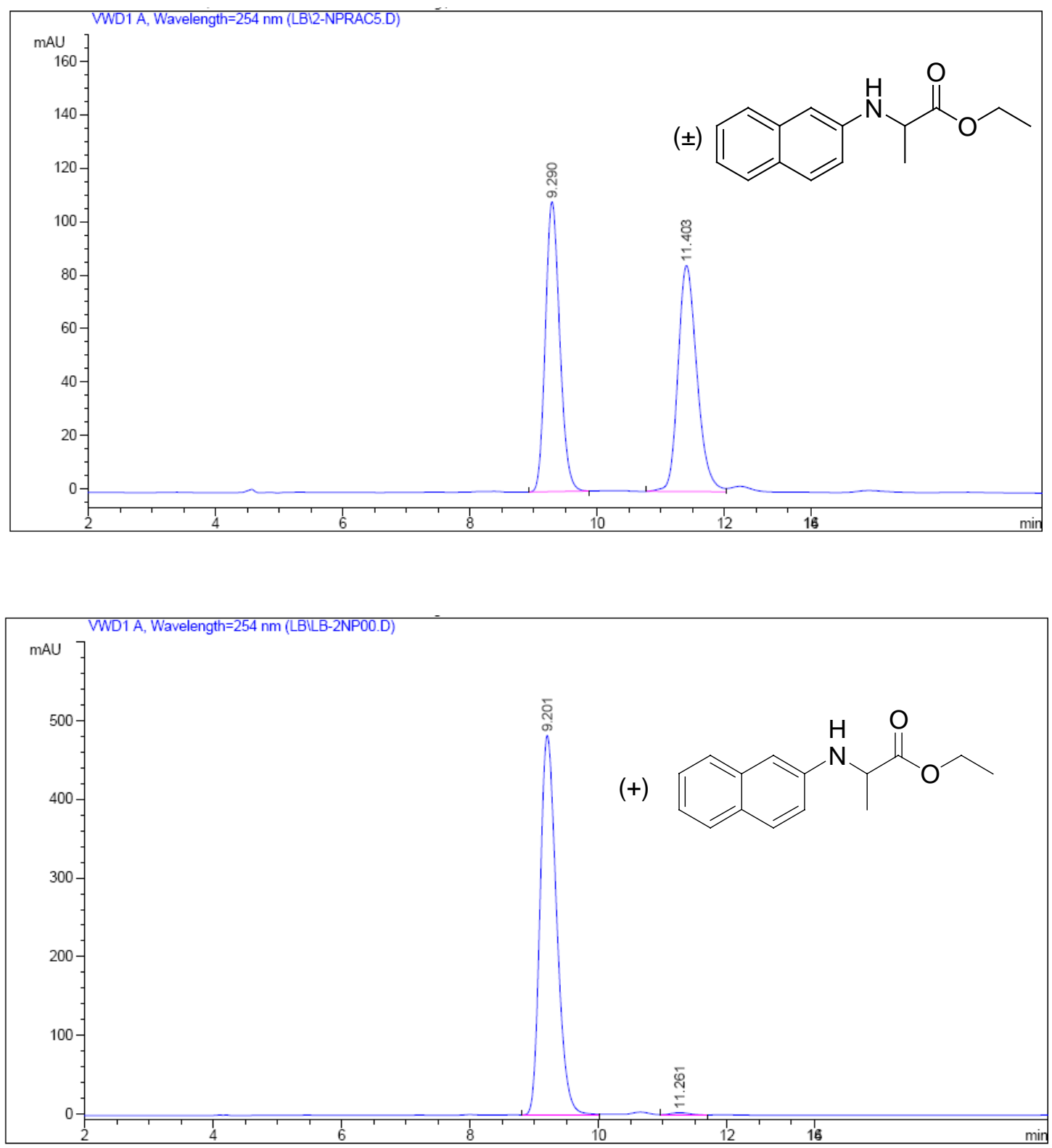

Signal 1: VWD1 A, Wavelength=254 nm

$\begin{gathered}\text { Peak RetTime Type } \\ \text { \# }\end{gathered}$ Width
[min]


(+)-Methyl 2-(phenylamino)propionate (4n)
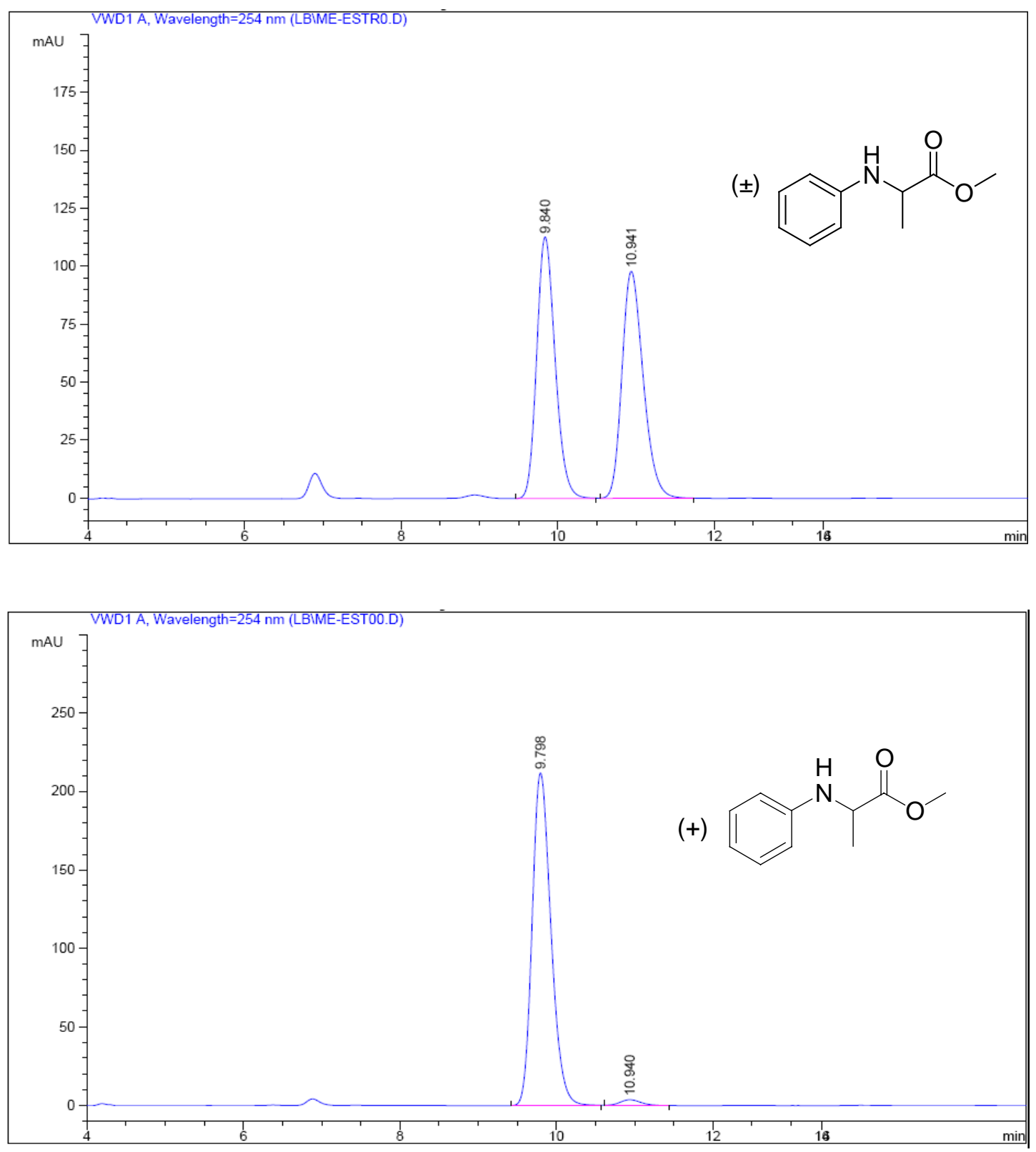

Signal 1: VWD1 A, Wavelength=254 nm

\begin{tabular}{|c|c|c|c|c|c|c|}
\hline Peak & RetTime & Type & Width & Area & Height & Area \\
\hline \# & [min] & & [min] & $\mathrm{mAU} \quad{ }^{*} \mathrm{~s}$ & {$[\mathrm{mAU} \quad]$} & 88 \\
\hline 1 & 9798 & $R P$ & -----1 & $\begin{array}{l}---1-1-1-1 \\
353363672\end{array}$ & 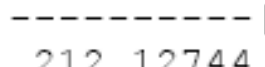 & -------1 \\
\hline 2 & 10.940 & $\mathrm{BB}$ & 0.2926 & 70.12914 & 3.67348 & 1.9460 \\
\hline
\end{tabular}


(+)-tert-Butyl 2-(phenylamino)propionate (4o)
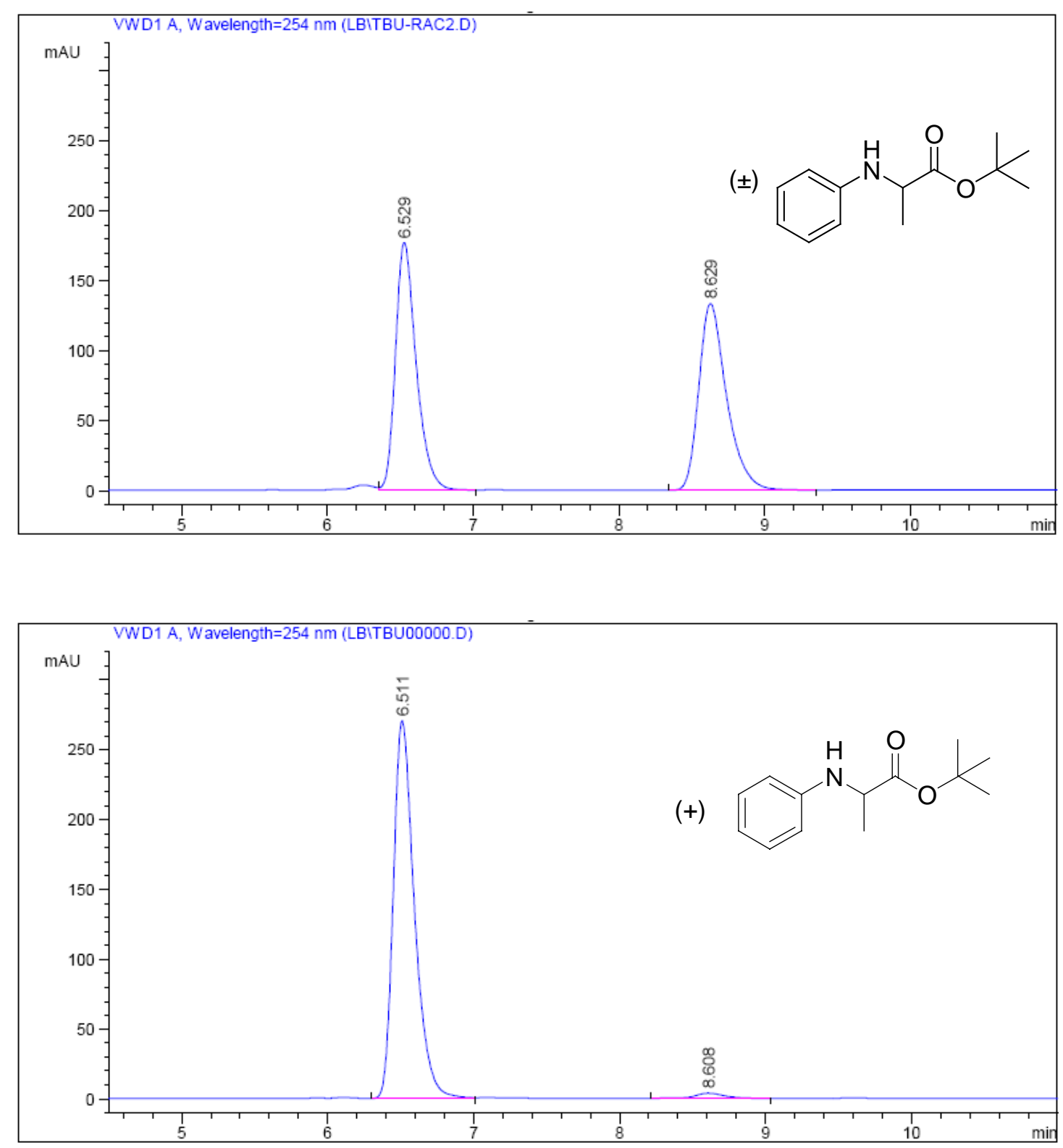

Signal 1: VWD1 A, Wavelength=254 nm

\begin{tabular}{|c|c|c|c|c|c|c|}
\hline Peak & RetTime & Type & Width & Area & Height & Area \\
\hline$\#$ & [min] & & [min] & $\mathrm{mAU} \quad{ }^{\star} \mathrm{s}$ & {$[\mathrm{mAU}]$} & the \\
\hline 1 & 651 & $\mathrm{VV}$ & 0.15 & 2711.87036 & 8592 & 0637 \\
\hline 2 & 8.608 & BP & 0.2111 & 53.54787 & 3.73179 & 1.9363 \\
\hline
\end{tabular}




\section{(+)-Ethyl 2-(phenylamino)butyrate (4p)}
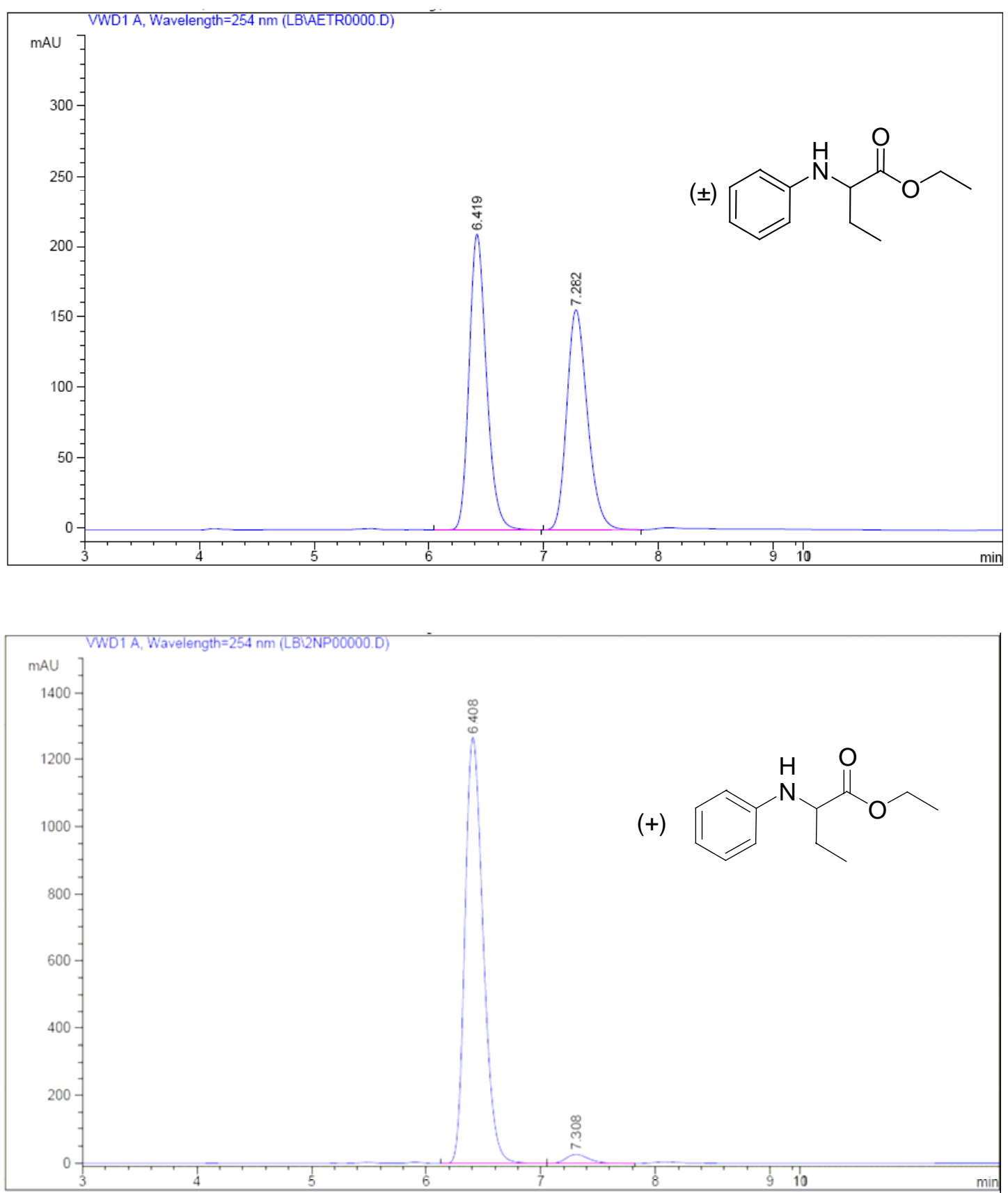

Signal 1: VWD1 A, Wavelength=254 nm

\begin{tabular}{|c|c|c|c|c|c|c|}
\hline \multirow{2}{*}{$\begin{array}{c}\text { Peak } \\
\#\end{array}$} & \multirow{2}{*}{$\begin{array}{c}\text { Retrime } \\
\text { [min] }\end{array}$} & \multirow{2}{*}{ Type } & \multirow{2}{*}{$\begin{array}{l}\text { Width } \\
\text { [min] }\end{array}$} & Area & Height & \multirow{2}{*}{$\begin{array}{c}\text { Area } \\
\frac{8}{8}\end{array}$} \\
\hline & & & & $\mathrm{mAU} \quad{ }^{*} \mathrm{~s}$ & {$[\mathrm{mAU} \quad]$} & \\
\hline & & 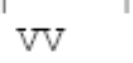 & & 1.40023 & & \\
\hline 2 & & $\mathrm{~V}$ & 07 & 400.95395 & 27.56659 & 2.7838 \\
\hline
\end{tabular}


(+)-Methyl 2-phenyl-2-(phenylamino)acetate (4q)
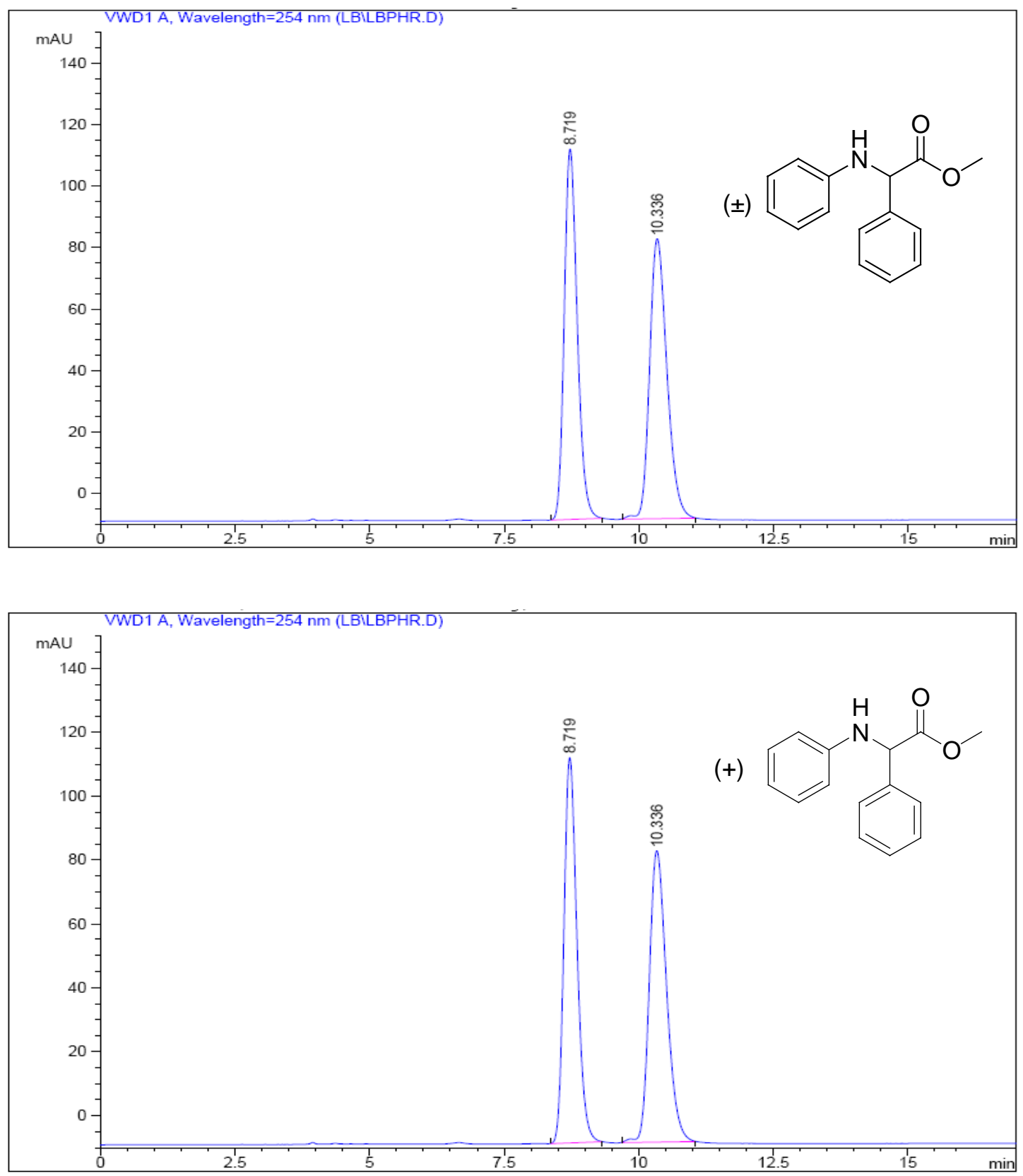

Signal 1: VWD1 A, Wavelength=254 nm

\begin{tabular}{|c|c|c|c|c|c|c|}
\hline Peak & Retrime & Type & width & Area & Height & Area \\
\hline & [min] & & {$[\min ]$} & $\mathrm{mAU} * \mathrm{~s}$ & {$[\mathrm{mzU} \quad]$} & 8 \\
\hline 1 & & $\mathrm{BB}$ & .2551 & 1169.48169 & 70.89153 & 54.0120 \\
\hline & & & & 995.74396 & 45.92213 & 45.9880 \\
\hline
\end{tabular}

